\title{
Mechanism of User Participation in Co-creation Community: A Network Evolutionary Game Method
}

\author{
Fanshun Zhang $\mathbb{D}^{1},{ }^{1}$ Congdong $\mathrm{Li} \mathbb{D}^{\mathrm{D}},{ }^{1}$ and Cejun Cao $\mathbb{D i D}^{2}$ \\ ${ }^{1}$ School of Management, Jinan University, Guangzhou 510632, China \\ ${ }^{2}$ School of Management Science and Engineering, Chongqing Technology and Business University, Chongqing 400067, China
}

Correspondence should be addressed to Congdong Li; licd@jnu.edu.cn and Cejun Cao; caocejun0601@tju.edu.cn

Received 15 November 2020; Revised 17 December 2020; Accepted 21 December 2020; Published 12 January 2021

Academic Editor: Baogui Xin

Copyright (c) 2021 Fanshun Zhang et al. This is an open access article distributed under the Creative Commons Attribution License, which permits unrestricted use, distribution, and reproduction in any medium, provided the original work is properly cited.

\begin{abstract}
Active participation closely associates with the sustainable operation of co-creation communities. Different from recent studies on the promotion of sustainable operation by identifying the internal and external motivations of user participation, this paper aims to analyze the mechanism regarding how different motivations affect the decision of user participation from group-level perspective. To better understand the mechanism, internal and external motivations are, respectively, captured by return-cost analysis and user interactive network. Afterwards, a network evolutionary game model was formulated to analyze the dynamic strategy selection (e.g., active participation and passive participation) of all users. In addition, the stable equilibrium and evolutionary path of strategies are analyzed through computational experiments. Results indicate the following: (a) Rewards have an influence on the promotion of active participation. However, with the continued growth of rewards, this promotion does not make sense sustainably. (b) The promotional effect of information noise on the selection of active participation can be found when passive participation is the dominant strategy. However, the inhibitory effect can be seen in populations that mainly adopt active participation. (c) The scale-free feature of user interactive network inhibits the selection of active participation when active participation is the dominant strategy in populations. Results found here is beneficial for managers to implement the specified policies and thus to achieve the sustainability of co-creation community.
\end{abstract}

\section{Introduction}

Co-creation community has significantly changed the role of users by breaking up the difference between producers and customers [1] and brought greater opportunities for promoting revenues and profits of companies [2]. About $60 \%$ of the world's biggest companies use online communities, and they allow users to share experiences and contribute creativity for product/service improvement [3]. Since every user acts as a content creator in co-creation communities, usergenerated contents play a critical role in product/service innovation [4]. However, many co-creation communities fail to survive because of the insufficient user participation [5]. Consequently, the key challenge for managers is to encourage user participation and to create a thriving community $[4,5]$.
User participation, which is defined as the extent to which users actively engage in the community's activities and interact with other users [6], is an emerging research topic in the field of marketing science [7] and information management systems [8]. Based on the definition of [6], users in co-creation communities can be divided into two types: passive users and active users. The former receives useful knowledge from communities without shared behavior. The latter is glad to share their experiences, ideas, and intelligences in a voluntary way, which plays an indispensable role in performance improvement of product innovation and profit making. However, Nielsen [9] portrayed that participation inequality was concluded as the result of the $90-9-1$ rule. $90 \%$ of users are lurkers (e.g., they read or observe, but they do not contribute). $9 \%$ of users contribute occasionally. $1 \%$ of users actively participate and account for 
most contributions. Thus, extensive studies on motivational identification of user participation were discussed from both internal and external perspectives, to promote active participation and thus to achieve the sustainability of co-creation community.

Based on the theory of personal behavior (e.g., commitment theory) and social behavior (e.g., social interaction theory), studies on internal aspects explained the motivation of user participation according to some views from psychological science and social science [4]. For example, Xu and $\mathrm{Li}$ [8] examined the influence of extrinsically oriented motivation (e.g., reputation and reciprocity) and intrinsically oriented motivation (e.g., altruism and enjoyment) on user participation. Zhou [10] tested the effect of social interaction (e.g., compliance and identification) on user participation. Tsai et al. [6] defined community participation as the interaction among members and their activity involvement, and several influential factors were analyzed from individual aspect (e.g., extraversion and need for affiliation), group aspect (e.g., identification and perceived critical mass), and relationship aspect (e.g., relationship satisfaction and relational trust). Roberts et al. [11] divided motivations of user participation into three aspects: motivations of innovation (e.g., interesting, passion, and skill development), motivations of contributing to community innovation activities (e.g., recognition, feedback, and ties to community) and motivations of directly collaborating with companies (e.g., desire for better products, career opportunities and economic goals). Chen et al. [12] discussed the intention of future participation from a perspective of knowledge sharing. They verified that knowledge sharing behavior had a great influence on user participation via customer learning value, socially integrative value, and hedonic value. On the other hand, a few studies concentrate on external motivations that relate to the external environment of communities. For example, Fang and Zhang [13] found that the network size which closely associated with number of users had a great influence on lurkers' participation. Based on the extensive studies on motivation of user participation, Malinen [14] concluded that group processes also affected online participation as well. For example, individuals (especially those with similar interests) associating with each other have a great influence on their decision.

Although many empirical studies were conducted to identity motivation of user participation, valuable topics should be further discussed on three aspects:

Firstly, motivations identified by researchers are based on questionnaire investigation for individual users, which ignores user interaction in co-creation communities. Basically, users build their interactive networks by publishing and responding to posts, and users who directly interact with each other over this network can be motivated to participate [15]. For example, user interaction was treated as group-level variables to analyze the influence of the interactive network on user participation [16]. Secondly, the initial motivations were identified by lots of researchers based on the assumption that the process of user participation is monocyclic and static [17]. However, user participation is a dynamic process in which users need to continuously decide whether to actively participate and when to actively participate next time. Users may choose active participation at present, adopt a passive strategy later, and use a combined strategy in the end. In this process, the decision is influenced by diverse motivations, and the influence of different motivations on user participation changes over time $[14,18]$. For example, free knowledge acquisition motives active participation at first, while rewards attract users to select the enduring active participation. Thirdly, despite the significant contributions made by existing studies on motivation identification, analyses of a mechanism regarding how different motivations influence user participation are still scarce in these studies. In other words, a mechanism regarding whether to actively participate, when to actively participate, and how such a strategy can perform well has not been established. As [17] argued, an analytical and mathematical method is needed to study the mechanism by using controlled variables and so as to understand the impact of various motivations on user participation. This work will bring opportunities for prediction of user participation behavior and creation of personalized services for different users [19]. A similar viewpoint of mechanism design based on different motivations was also suggested by [20].

Based on statements previously mentioned, the research question of this paper is how to formulate an adaptive model involving user interaction, dynamic decision-making processes, and motivations of users to analyze the mechanism regarding how different motivations affect the decision of user participation from group-level perspective. Fortunately, the network evolutionary game approach provides a powerful tool to explain the emergency of the given strategies (e.g., active participation and passive participation) in structural populations.

Three main tasks are implemented. Firstly, internal motivations (e.g. rewards, reputations, time costs and knowledge spillovers) are treated as returns and costs of users in the decision-making processes of user participation. A traditional evolutionary game model (TEGM) based on return-cost analysis is developed to analyze the stably evolutionary strategies (e.g. active strategy, passive strategy, or mixed strategy) between two individual users who are randomly selected from co-creation communities. Secondly, to integrate user interaction into the dynamic decisionmaking processes, a network evolutionary game model (NEGM) is applied to analyze the strategy selection of all users who are connected by a user interactive network. In this model, not only the effect of internal motivations but also the influence of external motivations (e.g., network size, network structure, and information noise) can be well discussed. Thirdly, since the evolution of user participation behavior has non-linear features, computational experiments are used to model the dynamic decision-making processes. The strategy evolution of user participation is presented by computational experiments.

The rest of this paper is organized as follows. Section 2 presents a critical literature review. Section 3 introduces the TEGM and the NEGM. The computational experiments are implemented in Section 4. Finally, conclusions and future directions are discussed in Section 5. 


\section{Literature Review}

The relevant studies are reviewed from three angles: (a) user participation in co-creation community; (b) modeling user participation through an evolutionary game model in networks; and (c) discovering the dynamic processes of strategy selection via computational experiments.

\subsection{User Participation in Co-Creation Community. Since} user participation closely associates with the sustainability of co-creation community, it has received a particularly growing attention in recent years. For example, Kohler et al. [21] highlighted the importance of experience in encouraging active participation, and they found that active participation was enhanced by enjoyable experiences (e.g., inspiring, intrinsically motivating, involving, and funny). Akman et al. [22] divided co-creation activities into four types, and they examined a mechanism regarding why users contributed their creativities in multiple activities. Based on different styles of employee communication, Lee and van Dolen [23] tested the influence of individual user sentiment on user behavior (e.g., creative behavior and community participation). To explore the reasons for volunteer participation in value co-creation activities, Lee and Kim [24] employed expectancy-value theory and empirically identified that three customer benefits (e.g., cognition, social integration, and hedonism) significantly affected their intentions of constant participation. According to the Stimulus-Organism paradigm, Kamboj and Rahman [4] developed a theoretical model to examine antecedents of branding co-creation from the perspective of user participation. Different from many studies focusing on motivational identification, Chepurna and Criado [25] tried to identify the barriers of value co-creation. Based on motivational aspects and deterrent identification, they developed a theoretical model to understand user participation behavior. Shen et al. [26] found that behavior of value co-creation was achieved by the interactivity of online community and online trust. Chen et al. [27] proposed a knowledge-sharing model to illustrate the reason why users choose to share their experiences for cocreation activities.

In summary, the literature mentioned onwards discussed user participation or user behavior in co-creation community from different perspectives. Table 1 presents the summary of these relevant studies. Five parts, including research question, motivational types, decision-making process, consideration of user interaction, and research method, are detailed to conclude the research focus of recent studies. In particularly, motivated by [4], motivations for user participation can be divided into two categories. On the one hand, internal motivation closely associates with the cognition of individual users (e.g., self-development, interest, social communication, and a sense of belonging to a social group). On the other hand, external motivation refers to external environments (e.g., network size, network structure, and information noise). As Jiang et al. [18] portrayed, recent studies assumed that the influence of initial motivation sustained for a long time, and users did not change their decisions over a longer time span. However, users' motivations often change over time, and users will change their strategies all the time. Therefore, the decisionmaking process is divided into two types: static process and dynamic process. Shen et al. [26] argued that online interaction was the critical antecedent of value co-creation behavior. The perceived interactivity, such as perceived quality and variety of information sharing, had a positive influence on active participation in co-creation activities via online trust. Therefore, user interaction is given extra consideration in the decision-making process of user participation in co-creation community. Research method is a suitable artifact (e.g., theoretical model, empirical research, and mathematical model) to contributes their main ideas.

From Table 1, user (continued) participation is a hot topic which is widely discussed in many papers. Based on the assumption of a static decision-making process, lots of researchers employed empirical methods to examine the influence of internal motivations on user participation from the investigation of individual users. However, few studies have investigated a mechanism of user participation (which also can be understood as a strategy selection of active participation or passive participation). In other words, there is a dearth of research directed towards analyzing the question concerning how user interaction influences the decision of user participation. Besides, a mathematical model for analyzing the dynamic decision-making processes is absent [14]. Thus, how to employ a mathematical model characterizing user participation as a dynamic decisionmaking problem is the special contribution of this paper.

2.2. Modeling User Participation through an Evolutionary Game Model in Networks. User participation behavior is a multiperiod decision-making process which users may continuously change their strategy based on different internal and external motivations from time to time. Vassileva [20] suggested that the mathematical model based on gametheoretical analysis was a good method for modeling this process. Particularly, as a popular method for modeling social interaction, TEGM provides an effective decisionmaking guidance for players with multiperiod games $[28,29]$. TEGM assumes that players have bounded rationality (e.g., players are reluctant to acknowledge complete information of others). For the first game with new players, they will make decisions based on the evaluation of their returns and costs. Next, they will adjust their strategies based on their previous actions (especially the successful strategy). Replicator dynamics, which is denoted by differential equation, describes the dynamic frequency change of the discrete decision in evolutionary games.

Based on different motivations, users may choose active participation at present, adopt a passive strategy later, and use a combined strategy in the end. Different motivations can be transformed in accordance with the returns and costs of users in multiperiod games. All users will adjust their strategies to maximize their returns (e.g., knowledge acquisition, reputation improvement, and satisfaction from 
TABLE 1: Summary of recent studies on user participation in co-creation community.

\begin{tabular}{|c|c|c|c|c|c|c|c|c|c|c|}
\hline \multirow[t]{2}{*}{ Study (year) } & \multirow[t]{2}{*}{ RQ or RO } & \multicolumn{2}{|c|}{$\begin{array}{c}\text { Types of } \\
\text { motivations }\end{array}$} & \multicolumn{2}{|c|}{$\begin{array}{c}\text { Decision-making } \\
\text { process }\end{array}$} & \multicolumn{3}{|c|}{ User interaction } & \multicolumn{2}{|c|}{$\begin{array}{c}\text { Research } \\
\text { method }\end{array}$} \\
\hline & & Internal & External & Static & Dynamic & Yes & No & $\mathrm{TM}$ & ER & MM \\
\hline Reference [21] (2011) & Attracting sustainable engagement & $\checkmark$ & & $\checkmark$ & & & $\checkmark$ & & $\checkmark$ & \\
\hline Reference [22] (2019) & Sustainable participation & $\checkmark$ & & $\checkmark$ & & & $\checkmark$ & & $\checkmark$ & \\
\hline Reference [23] (2015) & Creativity or participation & $\checkmark$ & & & $\checkmark$ & & $\checkmark$ & & $\checkmark$ & \\
\hline Reference [24] (2018) & Continued user participation & $\checkmark$ & & $\checkmark$ & & & $\checkmark$ & & $\checkmark$ & \\
\hline Reference [4] (2017) & Intention to active participation & $\checkmark$ & & $\checkmark$ & & & $\checkmark$ & & $\checkmark$ & \\
\hline Reference [25] (2018) & Barriers identification of co-creation & $\checkmark$ & $\checkmark$ & $\checkmark$ & & $\checkmark$ & & $\checkmark$ & & \\
\hline Reference [26] (2020) & Intention for co-creation activities & $\checkmark$ & $\checkmark$ & $\checkmark$ & & & $\checkmark$ & & $\checkmark$ & \\
\hline Reference [27] (2018) & Sustainable contribution & $\checkmark$ & & $\checkmark$ & & & $\checkmark$ & & $\checkmark$ & \\
\hline Reference [6] (2012) & Promotion of participation & $\checkmark$ & & $\checkmark$ & & & $\checkmark$ & & $\checkmark$ & \\
\hline Reference [11] (2014) & Engagement in innovation & $\checkmark$ & & $\checkmark$ & & $\checkmark$ & & $\checkmark$ & & \\
\hline Reference [13] (2019) & User continued participation & $\checkmark$ & $\checkmark$ & $\checkmark$ & & & $\checkmark$ & & $\checkmark$ & \\
\hline This paper & Mechanism of user participation & $\sqrt{ }$ & $\checkmark$ & & $\checkmark$ & $\checkmark$ & & & & $\checkmark$ \\
\hline
\end{tabular}

social exchange) in each game. Based on statements mentioned previously, TEGM provides a useful tool for modeling return-cost-based game between two players who are randomly selected from co-creation communities. However, user interaction, which is formulated by user-interactive behaviors (e.g., publish a post and respond to a post), is hard to be well analyzed in this model. Besides, it is also difficult to describe the influence of external environments on user participation. Therefore, researchers attempt to find another appropriate method to model user participation behaviors.

Fortunately, as an extensive method of TEGM, NEGM characterizes user interaction and dynamic decision-making process as a multiagent game model. This method was initially employed to analyze human cooperation in the field of physical science. NEGM fills up the gaps in which some factors (e.g., user interaction and the influence of external environment) are ignored in TEGM [30]. Particularly, user interaction is captured by a user interactive network where the connected edges indicate the presence of user interaction. Two-player game in NEGM generally occurs in two players who are connected by the given user interactive network. Besides, the consideration of user interaction is beneficial to examine the influence of external motivations (e.g., group preference, number of users and information noise caused by external environments) on user participation behaviors. Specially, group preference can be interpreted as the scale-free characteristic in complex networks. The number of users can be captured by the network size.

In recent years, NEGM has been widely used in the application of multiagent systems. For example, Esmaeilyfard et al. [31] developed a game-theoretical model to analyze user participation in ephemeral social vehicular networks. Al-Dhanhani et al. [17] employed a multiagent game model to discuss human cooperation in social applications under four circumstances (e.g., traditional Tit for Tat with fixed history, the generous Tit for Tat, reputationbased Tit for Tat, and group reputation-based Tit for Tat). Xu et al. [32] developed a multiagent game model for exploring user participation in collaborative filtering-based recommendation systems, and they found that the satisfactory recommendation depended on a high expectation of recommendation quality. Liu et al. [33] characterized patent cooperation as a multiagent system, and they constructed an industry and university \& research model to analyze the strategy selection of all users. Jiang et al. [18] investigated the evolution of knowledge-sharing behavior via a multigame model. However, there is a dearth of research directed towards investigating user participation in co-creation community [20].

Accordingly, the focus of this paper (e.g., internal motivations, external motivations, dynamic decision-making process, and consideration of user interaction) can be well modeled by the mathematical method of NEGM.

\subsection{Discovering the Dynamic Processes of Strategy Selection via} Computational Experiments. In TEGM, the dynamic processes of strategy selection are easily addressed by listing average returns of each player, analyses of replicator dynamics, calculation of stable evolutionary equilibrium, and presentation of strategy selection path. However, user participation in co-creation community is not determined by a simple accumulation of all results held in a single period of time. Analyses of NEGM involve the formulation of user interactive network, the establishment of an evolutionary game model, and the setting of evolutionary rules (e.g., how to change user's strategy based on their previous actions) in user interactive network [30]. In other words, this process modeled by NEGM has a nonlinear characteristic, which is difficult to be solved by an analysis tool in TEGM.

Fortunately, the computational experiment is a useful tool to understand the complexity and dynamics of the user participation system. The dynamic decision-making process can be well analyzed by setting environments, defining a theoretical model, designing an algorithm to simulate a multiagent system, and identifying the influence of different parameters on the decision made by players. Therefore, this method is widely applied to studying user interaction and user behaviors in social communication [18].

Motivated by [18], a user participation system can be divided into four parts. First, the user interactive network is set as the environment which influences the strategy 
selection of all users. Second, TEGM is set as the theoretical model based on return-cost analysis to describe the game between two players who are randomly selected from populations. Third, the multi-agent system of user participation is developed to present the dynamic decision-making process including network setting, evolutionary rules, and TEGM. Fourth, the influence of different parameters (e.g., network structure and network size) on user participation can be analyzed based on the result of computational experiments.

\section{NEGM of User Participation in Co- Creation Community}

User participation in co-creation community is a decisionmaking process which many users combined by user interactive network make decision on whether to active-participate or passive-participate in communities. According to literature review, this process can be captured by a multiagent model which integrates micro individual behavior (e.g., game between two users) and macroscopic phenomenon (e.g., individual behavior influenced by user interaction) into a systematic framework. Next, the micro individual behavior is described by TEGM in Section 3.1. The macroscopic phenomenon is elaborated by NEGM in Section 3.2. The framework of this paper is presented in Figure 1.

3.1. TEGM of User Participation and Its Analysis. In this section, the individual behavior of all users is formulated as a two-players game. Problem description, model formulation, and model analysis are detailed as follows.

3.1.1. Problem Description. Individual behavior of user participation is a decision-making process which user needs to decide whether to active participate or passive participate in a cocreation community. Since user participates in communities via online communicating technology, users fail to know what strategies are chosen by other users and how many returns are obtained by other users. Thus, users usually make their decisions based on the return-cost analysis which is abstracted from internal motivations. Table 2 shows the measurement of returns and costs in this decision-making process.

On one hand, users can obtain returns from their participations. Specially, internal motivations (e.g., interest, making friends, and reputations) account for active participation, while passive participation is driven by free way of knowledge acquisition and social communications. Besides, rewards paid by communities motivate active participation in communities. On the other hand, users should pay some costs, such as time, relationship maintenance, and a little money paid for knowledge acquisition.

3.1.2. Model Formulation. To develop a suitable model for better understanding problem description, the basic assumptions are elaborated as follows:

Assumption 1. Given that there is no difference among all users, two players in TEGM are the same stakeholders. In other words, a symmetric TEGM is proposed here.

Assumption 2. When a user decides to participate in a community, two strategies regarding active participation and passive participation can be chosen. They will make decisions based on their return-cost analysis. To present a comprehensive overview of various factors, parameter setting is summarized in Table 3.

Assumption 3. Denote $x$ as the probability that user B chooses active strategy, and 1-x indicates the probability that user B chooses passive strategy. For user A, $y$ and 1-y respectively represent the probability of choosing active participation or choosing passive participation. Three interactive modes (e.g. \{active, passive\}, \{passive, passive\}, and \{active, active\}) between two players are obtained from game matrix (see Table 4). The best situation is that both two players simultaneously choose active strategy, which brings opportunities for sustainable operation of communities.

3.1.3. Model Analysis. Based on the literature review, replicator dynamics method [34] is employed to analyze the TEGM.

The expected returns of user B that adopts active strategy are

$$
U_{\mathrm{BA}}=y\left(R_{1}-C_{1}\right)+(1-y)\left(R_{1}-C_{1}+W\right) .
$$

The expected returns of user $B$ that adopts passive strategy are

$$
U_{\mathrm{BP}}=y\left(R_{2}-C_{2}\right) .
$$

The average expected returns of user B under the mixed strategies are

$$
\overline{U_{B}}=x U_{\mathrm{BA}}+(1-x) U_{\mathrm{BP}}=\left(C_{2}-W-R_{2}\right) x y+\left(R_{1}-C_{1}+W\right) x+\left(R_{2}-C_{2}\right) y .
$$

Similarly, the average expected returns of user A under

$$
\overline{U_{A}}=\left(C_{2}-W-R_{2}\right) x y+\left(R_{1}-C_{1}+W\right) y+\left(R_{2}-C_{2}\right) x .
$$

the mixed strategies are 


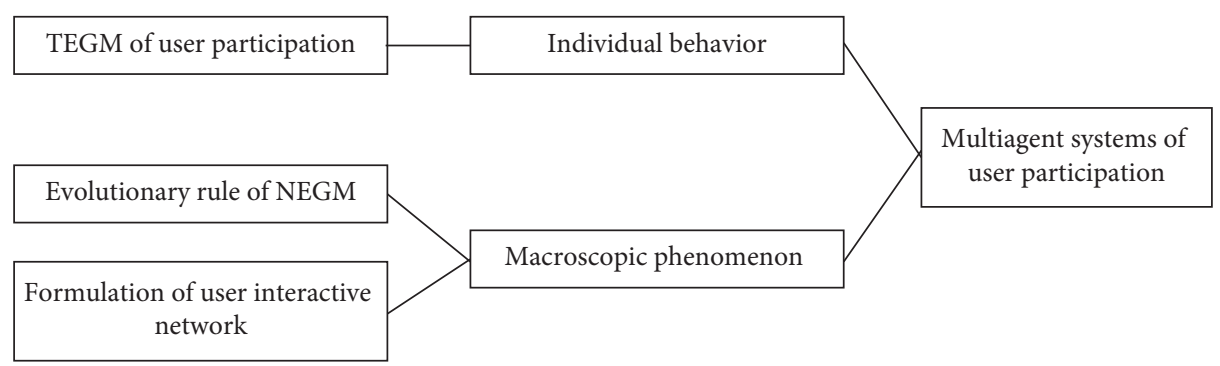

Figure 1: Framework of this paper.

TABLE 2: Measurement of returns and costs.

\begin{tabular}{lr}
\hline Items & Performance measurements \\
\hline Returns & Self-development, enjoyment, knowledge learning, recognition, making friends, empowerment, need for affiliation, \\
identification, relationship satisfaction, ties to community, reciprocity, reputation, passion, and other internal motivations \\
Costs & Lots of time, relationship maintenance, their own knowledge, a few moneys paid for knowledge acquisition \\
Rewards & Rewards from community, including tangible rewards and intangible rewards \\
\hline
\end{tabular}

TABLE 3: Parameter setting of TEGM.

$\begin{array}{ll}\text { Parameter } & \text { Description }\end{array}$

For users who passively participate in co-creation community

$R_{2}$ Returns obtained by passive users, such as knowledge spillover.

$\mathrm{C}_{2}$ Costs paid by passive users are their time, and few moneys paid for knowledge acquisition.

For users who actively participate in co-creation community

$R_{1} \quad$ Returns obtained by active users depend on feature of users. For example, narcissistic person prefers reputations from other users. Social enthusiasts concentrate on making friends, social communications, and relationship satisfaction.

$C_{1} \quad$ Costs paid by active users include their time, knowledge, and relationship maintenance.

W Rewards paid by co-creation community and free to acknowledge knowledge.

TABLE 4: Game matrix of user participation in co-creation community.

\begin{tabular}{llcc}
\hline & & User A & Passive $(1-y)$ \\
& & Active $(y)$ & $R_{1}-C_{1}+W ; R_{2}-C_{2}$ \\
User B & Active $(x)$ & $R_{1}-C_{1} ; R_{1}-C_{1}$ & $0 ; 0$ \\
\hline
\end{tabular}

According to the theory of TEGM, if the expected returns of a strategy selected by one user are greater than the average expected returns of populations, this strategy will spread in the population [28]. The frequency of such strategies can be described as the replicator dynamics which is denoted by the differential equation. Specially, the replicator dynamics system of user B is

$\frac{\mathrm{d} x}{\mathrm{~d} t}=x\left(U_{\mathrm{BA}}-\bar{U}\right)=x(1-x)\left[\left(R_{1}-C_{1}+W\right)+\left(C_{2}-W-R_{2}\right) y\right]$.

Similarly, the replicator dynamics system of user A is $\frac{\mathrm{d} y}{\mathrm{~d} t}=y\left(U_{\mathrm{AA}}-\bar{U}\right)=y(1-y)\left[\left(R_{1}-C_{1}+W\right)+\left(C_{2}-W-R_{2}\right) x\right]$.

Based on equations (5) and (6), five equilibrium points, namely, $(0,0),(1,0),(0,1),(1,1)$ and $\left(x^{*}, y^{*}\right)$, are obtained, where

$$
x^{*}=y^{*}=\frac{C_{1}-W-R_{1}}{C_{2}-W-R_{2}} .
$$

The stability of equilibrium points is obtained by analysis of the Jacobian matrix of replicator dynamics system. Based on [34], the Jacobian matrix is defined by equation (8):

$$
J=\left[\begin{array}{ll}
a_{11} & a_{12} \\
a_{21} & a_{22}
\end{array}\right]
$$

where

$$
\begin{aligned}
& a_{11}=(1-2 x)\left[\left(R_{1}-C_{1}+W\right)+\left(C_{2}-W-R_{2}\right) y\right], \\
& a_{12}=x(1-x)\left(C_{2}-W-R_{2}\right), \\
& a_{21}=y(1-y)\left(C_{2}-W-R_{2}\right), \\
& a_{22}=(1-2 y)\left[\left(R_{1}-C_{1}+W\right)+\left(C_{2}-W-R_{2}\right) x\right] .
\end{aligned}
$$

The determinant and the trace of Jacobian matrix are, respectively, denoted by $\operatorname{det} J$ and $\operatorname{tr} J$, where 


$$
\begin{aligned}
\operatorname{det} J & =a_{11} a_{22}-a_{12} a_{21}, \\
\operatorname{tr} J & =a_{11}+a_{22} .
\end{aligned}
$$

When $\operatorname{det} J>0, \operatorname{tr} J<0$, the equilibrium point is locally stable. In other words, the equilibrium point is the evolutionary stable strategy (ESS) of TEGM.

Based on the analysis mentioned onwards, three ESSs are discussed as follows:

Situation 1. If $R_{1}-C_{1}<R_{2}-C_{2}$ and $W<C_{1}-R_{1}$, ESS of group will reach "passive participation."

Situation 2. If $R_{1}-C_{1}<R_{2}-C_{2}$ and $W>C_{1}-R_{1}$, ESS of group will reach "active participation."

Situation 3. If $R_{1}-C_{1}<R_{2}-C_{2}$ and $W>\max \left\{C_{1}-R_{1}\right.$, $\left.C_{2}-R_{2}\right\}$, ESS of group will reach the mixed strategies of $(1,0)$ and $(0,1)$. It indicates that active and passive strategy are interchangeably chosen by users.

TEGM based on replication dynamics method is beneficial to analyze the multiperiod games of two players who are randomly selected from population and to design a mechanism of user participation in a microenvironment. However, evolutionary results of user participation are not a simple accumulation of all game states held in a single time period. Such evolutionary results, which are significantly influenced by the decision of other users, community's environment (e.g., group preference and number of users), and information noise, need to be considered for modeling user participation behavior in a macroscopic environment.

In sum, since the evolution has nonlinear features and evolutionary equilibrium points are hard to be calculated by TEGM, NEGM including game theory and complex network is considered next.

\subsection{NEGM of User Participation in Co-Creation Community.}

Once a user participates in a community, a user interactive network will be continuously enhanced via the created relationship with other users. The decision of user participation behavior is significantly influenced by user interactive network (e.g., group preference and number of users). For modeling user interaction and their game in networks, the step of NEGM formulation is detailed.

Step 1. Developing a network to present user interaction in a co-creation community. Specially, users who directly interact with the given user are called as the neighbors of the given user.

Step 2. At each time, all users calculate their returns based on the TEGM and compare with the returns obtained by their neighbors. Then, based on the evolutionary rules, they decide on whether to imitate neighbors' strategies.

Model assumptions, definition of network, and evolutionary rules are elaborated as follows.

3.2.1. Model Assumptions. Based on the properties of network observed by researchers focusing on co-creation community and the application of evolutionary game model, several assumptions are given.

Assumption 1. Since the focus of this paper is the connection among all users, the undirected network is represented by $G(V, E) . V=\left\{v_{i} \mid i=1,2, \ldots, n\right\}$ is a set of nodes (e.g., users in a co-creation community). $E=$ $\left\{e_{i j} \mid i, j=1,2, \ldots, n\right\}$ is a set of edges (e.g., user interactions in a co-creation community). $G$ is expressed by an adjacency matrix $A=\left(a_{i j}\right), a_{i j}=1$ indicates the presence of an undirected line, while $a_{i j}=0$ indicates the absence of an undirected line. Due to the undirected characteristic, there is no difference between $a_{i j}$ and $a_{j i}$. Assumption 2. All networks used in this paper are based on the simulated network. Community managers can adjust the parameter of the model to fit the realworld network and thus to comprehensively describe different situations in practice.

The characteristic of user interaction in co-creation community can be concluded from two aspects. On one hand, preferential attachment is the main principle of user interactive behavior. It indicates that users prefer to contact with persons with a high influence (e.g., leader users in co-creation community). Due to this feature, user interactive network generally emerges as a scale-free network [35]. On the other hand, users usually publish or respond posts within the given groups which persons have the same interests (e.g., improvement of products/services and feedback of using experiences). Such user behaviors lead to user interactive network consisting of many small groups. Specially, connections are dense within the small groups, while connections are sparser between different small groups [36].

To further verify the scale-free feature and the occurrence of small groups, the authors did an empirical study via real data which is collected from the famous co-creation community of Pollen Club. Pollen Club is a social networking platform for customers who use the electronic equipment (e.g., mobile phone) produced by Huawei. Users can publish posts to share their experiences, to suggest for product improvement, to ask for guidance, and to provide a feedback of phone using. For emphasizing the importance of co-creation, posts discussing suggestions for product improvement are considered. Huawei P40, launched at 21:00 p.m., March 26, 2020, is the newest mobile phone which receives a growing attention. To explore the evolution of users' behaviors from the beginning, the time period for this case is from March 26, 2020 to September 5, 2020. The data extracted from Pollen Club includes user name, time, and topic. Finally, 64302 posts, 8197 users' names as well as posting time are collected by the tool of Octopus Data Collector. Based on this data, user interactive network of $8197 * 8197$ is formulated by Python tool. According to [37], scale-free features and the 
characteristic of small group can be respectively proved by cumulative degree distribution and hierarchical organization (see Figure 2).

From Figure 2, empirical results provide a powerful verification that user interactive network in co-creation community has scale-free features and the occurrence of small groups.

To formulate the interactive network mentioned onwards, network generation model concerning the growth of users and user interactions is chosen. More details of the proposed model are discussed in the next section.

Assumption 3. Given that user participation behavior is significantly influenced by return-cost analysis of other users, the evolutionary rule (e.g. replicator dynamics) is chosen to update users' decisions. The strategy selection depends on the last game result, and the memory length is set to 1 .

Next sections introduce formulation of user interactive network and evolutionary rules.

3.2.2. Definition of User Interactive Network. Based on Assumption 2, the evolving network model proposed by [36] is employed to generate networks similar as the desired networks including scale-free features and the occurrence of small groups. The processes of network generation are elaborated.

Step 1 (Initialization). The initial network includes $M$ small groups where $m_{0}$ nodes are connected as the complete graph. $M$ nodes respectively selected from $M$ small groups are connected as the complete graph, which indicates that both two small groups are connected by the initial edge at least.

Step 2 (Growth). At each time $t$, a new node participates in a network and joins in a small group $j$. Then, the new node connects $m\left(m<m_{0}\right)$ nodes within group $j$ through inner-group links. Meanwhile, the new node connects $n(n<m)$ nodes in other $M-1$ groups through inter-group links with a probability of $\alpha(0<\alpha<1)$. For taking account preferential principle into the network generation model, the interactive attachments are concluded:

(a) Inner-group preferential attachment. The probability $\left(P\left(s_{i j}\right)\right)$ that a new node connects node $i$ in small group $j$ depends on the degree $s_{i j} . P\left(s_{i j}\right)$ is defined as

$$
P\left(s_{i j}\right)=\frac{s_{i j}}{\sum_{k} s_{j k}}
$$

where

$s_{i j}$ is the degree of node $i$ in group $j . k$ is the total number of nodes in group $j . \sum_{k} s_{j k}$ is the total degree of nodes in group $j$.

(b) Inter-group preferential attachment. The probability $\left(P\left(l_{i h}\right)\right)$ that a new node connects node $i$ in a small group $h(h \neq j)$ depends on the degree $l_{i h} . P\left(l_{i h}\right)$ is defined as

$$
P\left(l_{i h}\right)=\frac{l_{i h}}{\sum_{m, n, n \neq j} l_{m, n}},
$$

where $l_{i h}$ is the degree of node $i$ in the externa group $h(h \neq j) . n$ is the sum of external group (apart from group $j) . m$ is the number of nodes which connects external node in group $n(n \neq j) . \sum_{m, n, n \neq j} l_{m, n}$ is the sum of nodes which connect the external node.

Step 3 (Formulation). To generate the desired network mentioned onwards, the algorithm is given in Algorithm 1 .

3.2.3. Evolutionary Rules of NEGM. Based on Assumption 3, replicator dynamics method is chosen.

$J_{i}=\left\{j_{1}, j_{2}, \ldots, j_{k_{i}}\right\}\left(k_{i}\right.$ is the degree of node $\left.i\right)$ is a set of nodes which are connected by node $i$. In other words, $J_{i}$ is all neighbors of node $i$. At time $t, p_{i}(t)$ is the probability that a user actively participates in a community. The expected returns of node $i$ and node $j$ is calculated as

$$
\begin{aligned}
& U_{i}=\sum_{i \in I_{i}}\left(U_{\mathrm{BA}}^{i} * p_{i}(t)+U_{\mathrm{BP}}^{i} *\left(1-p_{i}(t)\right)\right)=\sum_{i \in I_{i}}\left(y *\left(R_{1}-C_{1}\right) * p_{i}(t)+(1-y) *\left(R_{1}-C_{1}+W\right) * p_{i}(t)+y *\left(R_{2}-C_{2}\right) *\left(1-p_{i}(t)\right)\right), \\
& U_{j}=\sum_{j \in J_{i}}\left(U_{\mathrm{BA}}^{j} * p_{j}(t)+U_{\mathrm{BP}}^{j} *\left(1-p_{j}(t)\right)\right)=\sum_{j \in J_{i}}\left(y *\left(R_{1}-C_{1}\right) * p_{j}(t)+(1-y) *\left(R_{1}-C_{1}+W\right) * p_{j}(t)+y *\left(R_{2}-C_{2}\right) *\left(1-p_{j}(t)\right)\right) .
\end{aligned}
$$

At time $t+1$, if $U_{i}>U_{j}$, the initial strategy is still chosen by node $i$. Otherwise, the decision of node $i$ evolves as the neighbor $j$ 's strategy. The probability $p_{i}(t+1)$ that a user actively takes part in a community is

$$
p_{i}(t+1)=p_{i}(t)\left(1-P_{s_{i}-s_{j}}\right)+p_{j}(t) P_{s_{i}-s_{j}}
$$

where $P_{s_{i}} \longrightarrow s_{j}$ is the probability that node $i$ imitate the strategy of user $j$, and it is defined as 


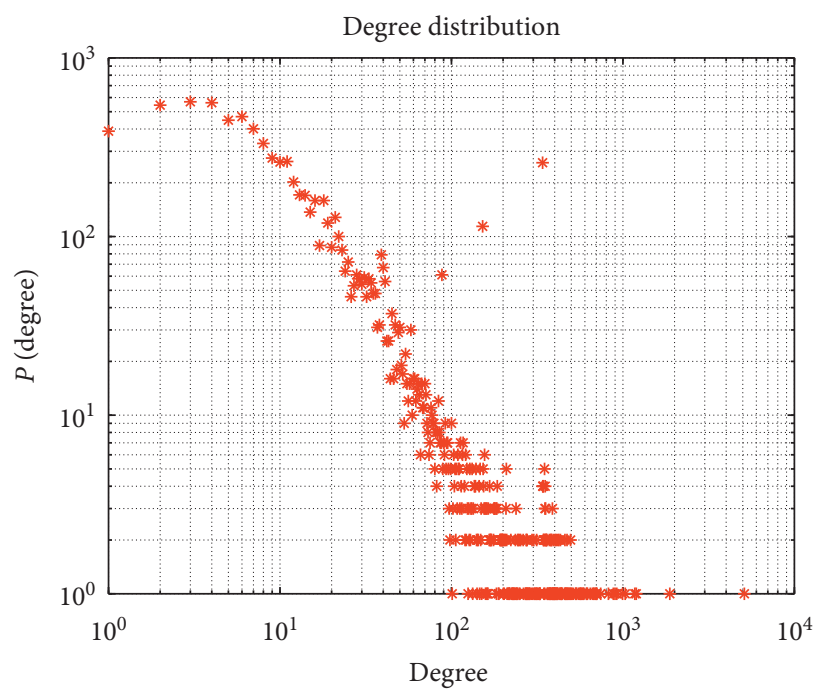

(a)

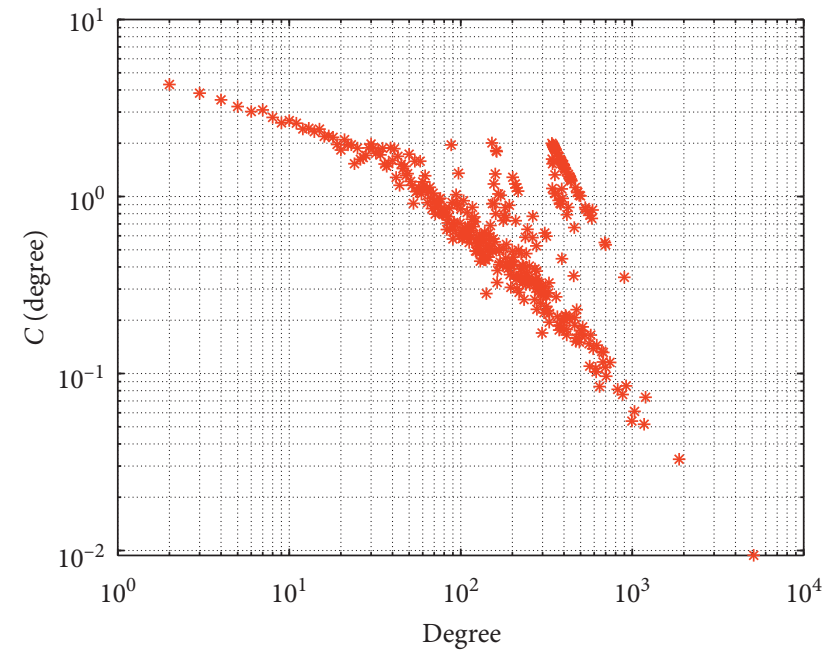

(b)

FIGURE 2: Result of cumulative degree distribution and hierarchical organization. (a) Cumulative degree distribution. (b) Hierarchical organization.

Input: The initial number of nodes $m_{0}$, the desired edges $N$ and the iteration number $t=N-m_{0}$, the number of additive edges $m$ within the inner group at each time $t$, the number of additive edges $n$ in external groups at each time $t$, the probability $\alpha$ that a new node connects the node in external groups, number of small groups $M$, and size of each small group Group_Size.

Initially: For $t=0, M * m_{0}$ number of nodes within the inner group are connected as a complete graph. $M$ number of nodes which are, respectively, selected from different groups are connected as a complete graph.

For $1 \leq t \leq N$, do

Step 1: Formulate the network $G_{t}$ based on equations (11) and (12).

Step 2: If $t<T$, go to step 1; otherwise, stop.

Output: $G_{t}$.

Algorithm 1: Network formulation of co-creation community.

$$
P_{s_{i} \longrightarrow s_{j}}=\frac{1}{1+e^{\left(\left(U_{i}-U_{j}\right) / \delta\right)}},
$$

where $\delta$ is the information noise in the decision-making process. $\delta \longrightarrow 0$ indicates that user $i$ is completely rational. Node $i$ is sure to imitate the strategy of $j$. When $\delta \longrightarrow \infty$, $P_{s_{i}} \longrightarrow s_{j}$ approximately equals $1 / 2$. It indicates that user $i$ is bounded rational. User $i$ study the strategy of node $j$ at random.

The strategy (e.g., active or passive participation) chosen by users and the related returns are the focus of this paper. Hence, denote $\bar{P}$ and $\bar{U}$ to, respectively, represent them.

$$
\begin{aligned}
& \bar{P}=\lim _{t \longrightarrow+\infty} \sum_{i} \frac{p_{i}(t)}{n}, \\
& \bar{U}=\lim _{t \longrightarrow+\infty} \sum_{i} \frac{U_{i}}{\bar{k} n} .
\end{aligned}
$$

\section{Computational Experiments of NEGM}

In this section, NEGM of user participation behavior is analyzed by some computational experiments. Specially, internal motivations (e.g., self-development, reputation, and rewards) are discussed by return-cost analysis presented in Section 4.1. External motivations (e.g., network size, information noise, and network structure) are discussed in Section 4.2.

Motivated by [18], the computational system is divided into four modules:

(a) Develop the network, and define the game parameters and model parameters.

(b) Graphically present the network evolution, and show the change of nodes and the created relationships.

(c) Display the results, and show the degree of a network.

(d) Draw the evolutionary strategies $(\bar{P})$ and the related returns $(\bar{U})$.

Computational experiments are implemented by Matlab R2018b. The Algorithm 2 is given next.

4.1. Computational Results Discussed from Internal Motivations. The impact of internal motivations on user participation can be understood as the influence of game parameter (e.g., returns of $R_{1}$ and $R_{2}$, costs of $C_{1}$ and $C_{2}$, 
Input: (1) Network setting: the graph $G_{t}$ generated by Algorithm 1; (2) the iteration number: $t=T$; (3) Evolutionary game model: returns of $R_{1}$ and $R_{2}$, costs of $C_{1}$ and $C_{2}$, reward $W$, the probability $p$ and $1-p$ for choosing active behavior or passive behavior; (3) Network properties: the degree of each node $k_{i}$.

Initially: For $t=0, M * m_{0}$ number of nodes within the inner group are connected as a complete graph. $M$ number of nodes which are, respectively, selected from different groups are connected as a complete graph. Next, denote $p_{i}(0)=0.5$ as the initial proportion that users choose active strategy.

For $1 \leq t \leq N$, do

Step 1: Calculate the expected returns of each node $U_{i}$ based on equations (13) and (14).

Step 2: For each node $i$, select a neighbor $j$ at random. Update $p_{i}(t+1)$ of node $i$ based on equation (15).

Step 3: Calculate $\bar{P}$ and $\bar{U}$ according to equations (17) and (18).

If $t<T$, go to step 1 ; otherwise, stop.

Output: $\bar{P}$ and $\bar{U}$

Algorithm 2: Simulation of NEGM.

reward $W$ ) on evolutionary strategies $(\bar{P})$ and the average returns $(\bar{U})$ in NEGM. Therefore, to provide a better comparison among different situations, parameters regarding external motivations are set as the same value in this section. Specially, network size is set as 2000 , which indicates that there are 2000 users in this community. User interactive network has scale-free feature and some small groups. To reduce the influence of network structure on decision of user participation behavior, number of small groups and size of each small group are, respectively, denoted as 4 groups and 500 users. Given that two-player games occur many times (e.g., when users take part in communities and scan posts published in community, they need to make decisions on whether to active or passive participate), the iteration time of evolutionary game $(t)$ is set as 500 times. To reduce the influence of information noise, $\delta$ is denoted as 100 . Next, different situations regarding the influence of internal motivations on their decisions are detailed .

Situation 1. When $R_{1}>C_{1}, R_{2}>C_{2}, R_{1}>R_{2}, C_{1}>C_{2}$, and $R_{1}-C_{1}>R_{2}-C_{2}$, both users choosing active strategy and users choosing passive strategy can obtain reasonable returns from their actions. Specially, returns obtained by active users are bigger than returns gained by passive users. This is the best situation of "more work for more pay." To describe this situation, parameters are set as $R_{1}=20, R_{2}=15, C_{1}=8$, and $C_{2}=6$. For observing the influence of rewards (W) on their decisions, five cases are, respectively, set as $0,3,6,9$, and 12 . Figure 3 depicts the evolutionary strategies path $(\bar{P})$ and average returns $(\bar{U})$. Hierarchical axes, respectively, represent $\bar{P}$ and $\bar{U}$ in Figures 3(a) and 3(b). Specially, $\bar{P}=1$ indicates that active participation is chosen by all users. $\bar{P}=0$ indicates that passive participation is chosen by all users. $\bar{P}=(0,1)$ indicates that the mixed strategies are chosen by users. Horizontal axis represents the iteration number of evolutionary game $(t)$.

From Figure 3(a), users' strategies evolve as active participation with a probability of 0.56 in a short time $(t=32)$, although there is no reward paid for active users. When community managers reward active users, the probability for choosing active strategies continuously increases. Specially, $w=12$ plays a significant role in promoting active participation (e.g., $\bar{P}$ reaches to 0.76). From Figure 3(b), average returns earned by users are located at interval $[8.3,12.9]$, which indicates that all users can obtain satisfied returns in this situation.

Situation 2. When $R_{1}>C_{1}, R_{2}>C_{2}, R_{1}>R_{2}, C_{1}>C_{2}$, and $R_{1}-C_{1}<R_{2}-C_{2}$, a bad situation of "less work for more pay" occurs, which indicates that passive users obtain excessive returns from volunteer behavior of active users. To present this situation, parameters are set as $R_{1}=16$, $R_{2}=12, C_{1}=13, C_{2}=6, W=[0,3,6,9,12]$. Figure 4 shows the results of $\bar{P}$ and $\bar{U}$.

From Figure 4(a), populations prefer to choose passive strategy $(1-\bar{P} \approx 0.53)$ without consideration of rewards, which results from the unreasonable return-cost allocation. From Figure 4(b), average returns continuously increase as the growth of rewards. However, the probability for choosing active participation does not increase all the time, although the increasing caused by the growth of rewards is obvious (e.g., compared with situation of $W=0$, other four situations considering rewards promote strategy selection of active participation). For example, $W=3$ promotes active participation, while $W=9$ inhibits active participation. This result differs from result discovered in TEGM, which indicates that rewards do not always promote active participation. According to [38], this phenomenon can be explained by the special network structure (e.g., scale-free feature). When a strategy is popular in a group, this strategy will spread in these populations. In other words, evolutionary strategies depend on the initial strategy which is spread in populations.

Situation 3. When $R_{1}>C_{1}$ and $R_{2}<C_{2}$, three possible cases including $\left\{R_{1}>R_{2}, C_{1}>C_{2}\right\},\left\{R_{1}>R_{2}, C_{1}<C_{2}\right\}$, and $\left\{R_{1}<R_{2}, C_{1}<C_{2}\right\}$ bring opportunities for the establishment of best situation regarding $R_{1}-C_{1}>$ $R_{2}-C_{2}$. To simulate these three situations and compare with them, parameter setting is denoted in Table 5. Figure 5 concludes three cases mentioned in situation 3 . 


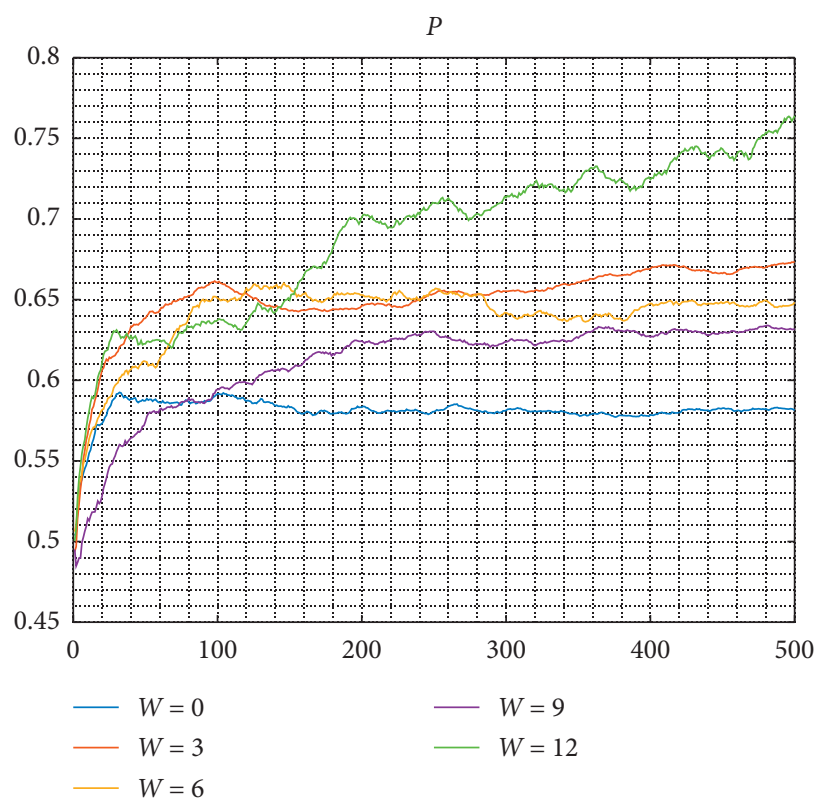

(a)

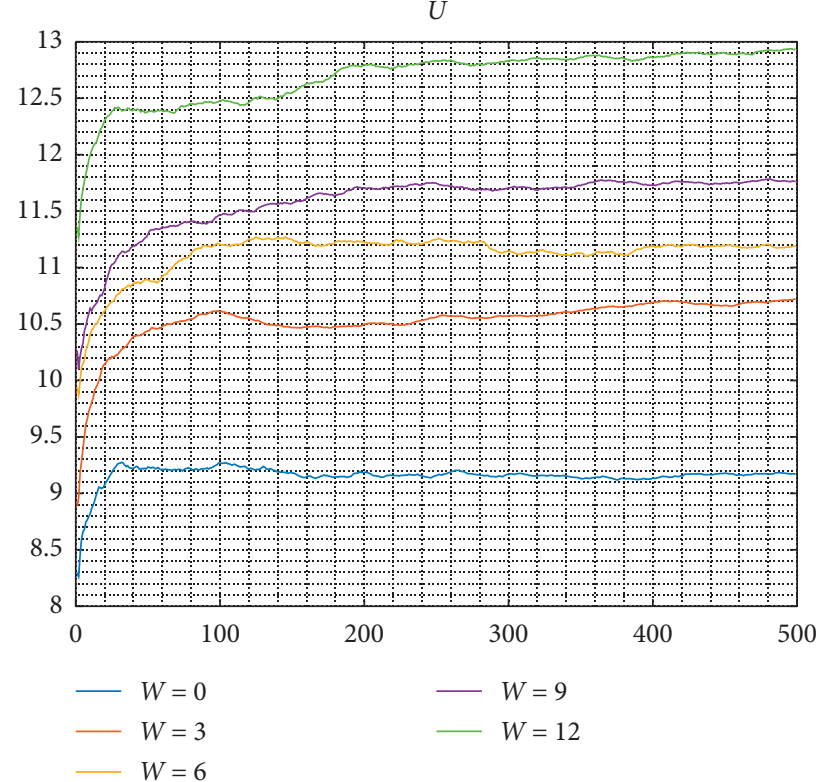

(b)

Figure 3: The simulated results of situation 1. (a) $\bar{P}$. (b) $\bar{U}$.

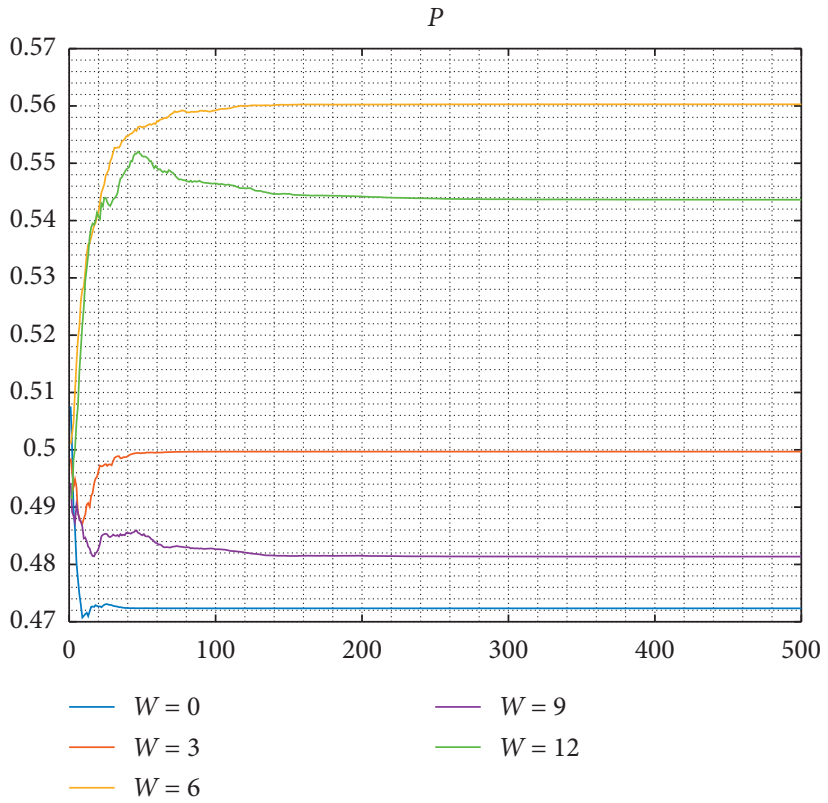

(a)

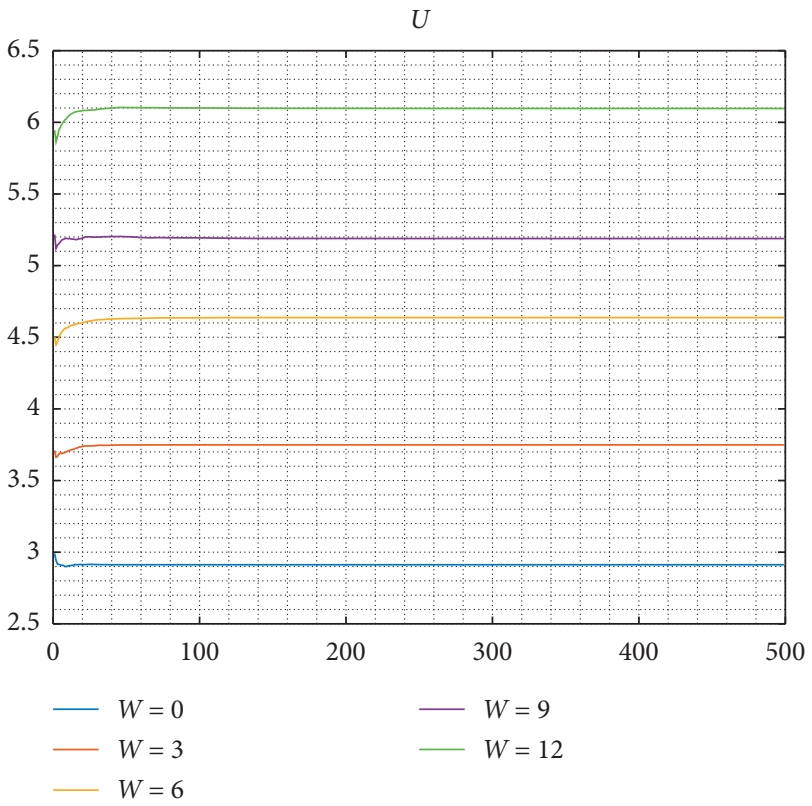

(b)

FIgURE 4: The simulated results of situation 2. (a) $\bar{P}$. (b) $\bar{U}$.

TABle 5: Parameter setting of situation 3.

\begin{tabular}{lccccr}
\hline & $R_{1}$ & $R_{2}$ & $C_{1}$ & $C_{2}$ & $W$ \\
\hline Case 1: $R_{1}>R_{2}, C_{1}>C_{2}$ & 18 & 6 & 10 & 8 & {$[0,3,6,9,12]$} \\
Case 2: $R_{1}>R_{2}, C_{1}<C_{2}$ & 22 & 10 & 10 & 12 & {$[0,3,6,9,12]$} \\
Case 3: $R_{1}<R_{2}, C_{1}<C_{2}$ & 16 & 18 & 13 & 20 & {$[0,3,6,9,12]$} \\
\hline
\end{tabular}


12

Complexity

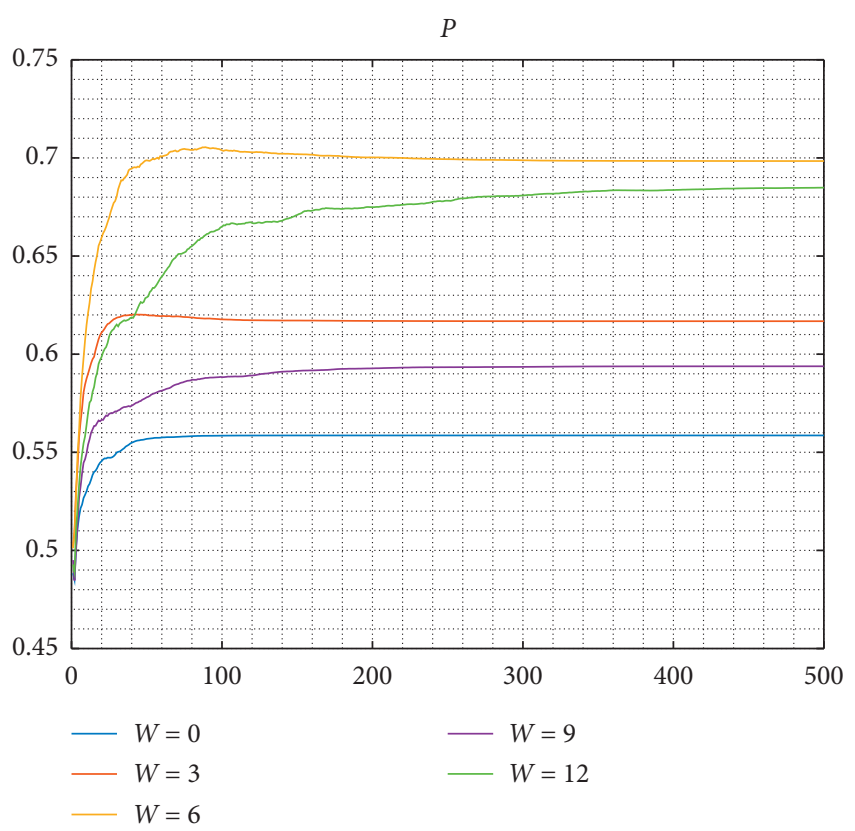

(a)

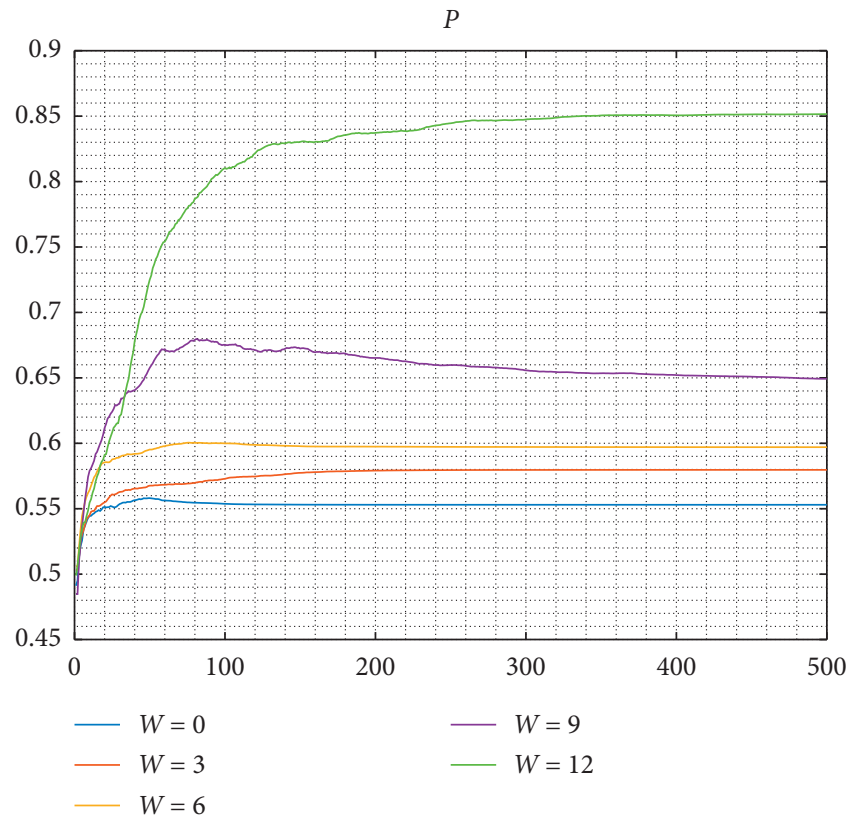

(c)

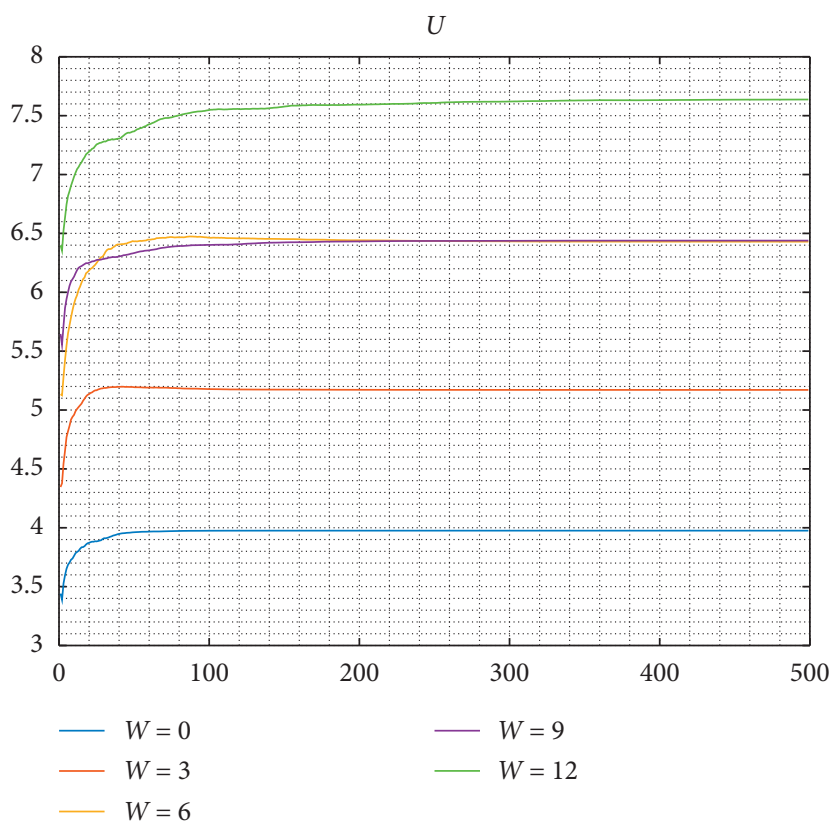

(b)

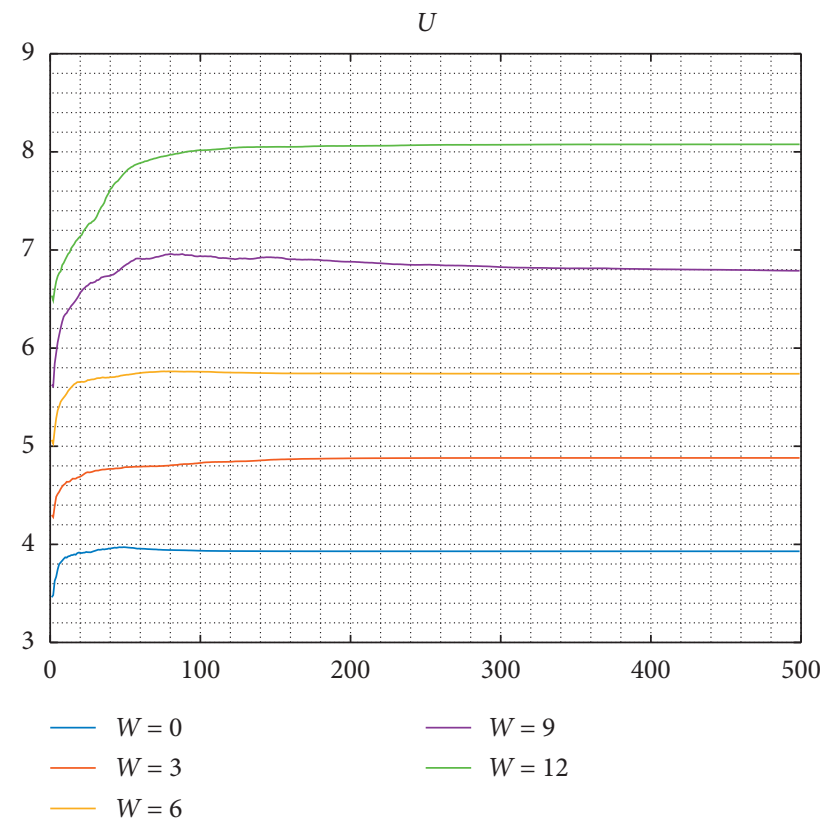

(d)

FIgure 5: Continued. 


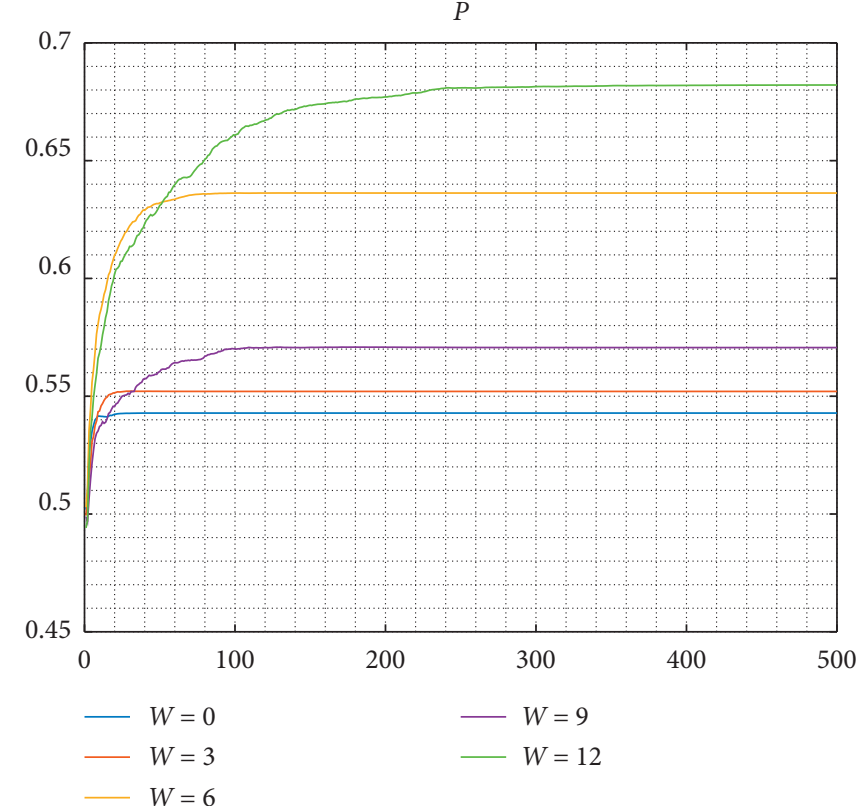

(e)

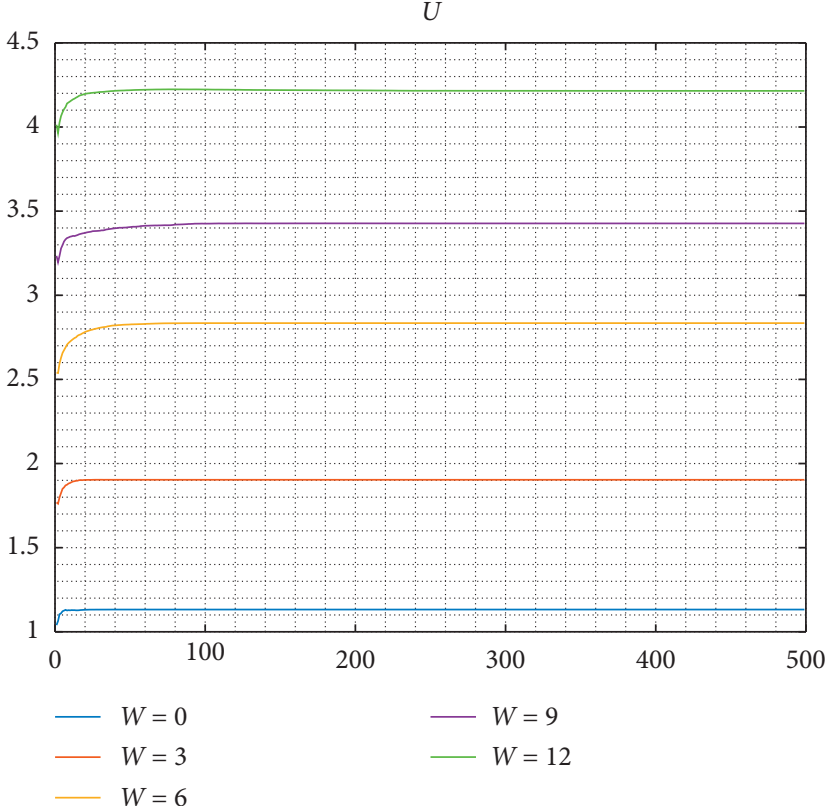

(f)

Figure 5: The simulated results of situation 3. (a) $\bar{P}$ (Case 1). (b) $\bar{U}$ (Case 1). (c) $\bar{P}$ (Case 2). (d) $\bar{U}$ (Case 2). (e) $\bar{P}($ Case 3 ). (f) $\bar{U}($ Case 3).

TABLE 6: Parameter setting of situation 4.

\begin{tabular}{lccccr}
\hline & $R_{1}$ & $R_{2}$ & $C_{1}$ & $C_{2}$ & $W$ \\
\hline Case 1: $R_{1}>R_{2}, C_{1}>C_{2}$ & 18 & 16 & 22 & 14 & {$[0,3,6,9,12]$} \\
Case 2: $R_{1}<R_{2}, C_{1}>C_{2}$ & 16 & 20 & 18 & 16 & {$[0,3,6,9,12]$} \\
Case 3: $R_{1}<R_{2}, C_{1}<C_{2}$ & 6 & 18 & 8 & 10 & {$[0,3,6,9,12]$} \\
\hline
\end{tabular}

From Figure 5, some observations are concluded:

(a) Due to $R_{1}-C_{1}>R_{2}-C_{2}$, users prefer to choose active participation $(\bar{P}>0.5)$ without considering rewards.

(b) Rewards sometimes promote active participation (e.g., red line in Figure 5) but sometimes inhibits active participation (purple line in Figures 5(a) and $5(\mathrm{~b}))$.

(c) Case 3 usually occurs in the maturation of community's operation. Rules (e.g., how many rewards should be paid for active users, how to encrypt the valuable knowledges which are contributed by active users, and how much money should be paid by passive users for acknowledging valuable knowledges) are well designed and implemented for a long time. These rules lead to expensive costs for users who passively participate in communities. And the rules also avoid the occurrence of knowledge spillover. In this context, to acknowledge valuable knowledges from community, passive users have no choice but to contribute their knowledges, experiences, and skills for exchange. (d) From Figures 5(b), 5(d), and 5(f), average returns are located at interval $[1,8]$. The value is much smaller than the value in the same situation of "more work for more pay" (situation 1). Situation 1 describes that both active users and passive users can obtain high returns from communities. This situation usually occurs in the communities which include lots of valuable knowledges and many active users. For example, valuable knowledges attract users to participate in communities, and they obtain returns from learning knowledges. Besides, many active users indicate that users obtain returns from social communications via interaction with other persons. In situation 3, the limited valuable knowledges created by active users will be locked by managers. Active users can easily obtain these knowledges. However, passive users need to pay some money for acknowledging knowledges. This situation results in the outcome that few returns can be obtained by passive users. Therefore, although situations 1 and 3 present the same situation of "more work for more pay", the influential path on the decision of user participation is different. 
Situation 4. When $R_{1}<C_{1}$ and $R_{2}>C_{2}$, three possible cases including $\left\{R_{1}>R_{2}, C_{1}>C_{2}\right\},\left\{R_{1}<R_{2}, C_{1}>C_{2}\right\}$, and $\left\{R_{1}<R_{2}, C_{1}<C_{2}\right\}$ lead to the worse situation of $R_{1}-C_{1}<R_{2}-C_{2}$. To simulate these three situations and compare with them, parameter setting is denoted in Table 6.

Figure 6 concludes three cases mentioned in situation 4 . From Figure 6, some observations are concluded:

(a) Due to $R_{1}-C_{1}<R_{2}-C_{2}$, users prefer to choose passive strategy $(1-\bar{P}>0.5)$ without consideration of rewards.

(b) Situation 4 relates to the worse situation of "less work for more pay", which indicates that returns earned by active users are smaller than returns obtained by passive users. This situation inhibits active participation and thus leads to nonsustainability of communities. From Figures 6(a), $6(\mathrm{c})$, and 6(e), rewards play a slight role in promoting active participation. Specially, the probability for choosing active strategy is bigger than 0.5 only when $W=12$.

(c) From Figures 6(b), 6(d), and 6(f), average returns are located at interval $[-1.4,3.9]$. This value is much smaller than the value generated by other situations. Particularly, Figure 6(b) shows that average returns are smaller than zero, which indicates that lots of users (especially active users) cannot obtain reasonable returns from their volunteer behavior (e.g., experience sharing, knowledge sharing and helping others). This situation often occurs in the early stage of community's operation or the recession of community's operation.

On the one hand, in the early stage of community's operation, only a few active users contribute their knowledges and experiences in the co-creation community. Other users act as lurkers to scan the knowledges and experiences. In this situation, community managers need to maintenance the current active users via rewards (e.g., financial benefits and non-economic rewards). Meanwhile, rewards should also be paid for passive users who attempt to choose active strategy.

On the other hand, in the recession of community's operation, managers should decide whether to set rewards for users. From Figures 6(a), 6(c), and 6(e), only when $W=12$, populations will choose active strategy with a probability of 0.5 . It indicates that lots of costs (e.g., money) should be paid to maintenance community's operation. In this situation, managers should evaluate the value of community for company, before they decide whether to pay more money for community's operation.

Situation 5. When $R_{1}<C_{1}, R_{2}<C_{2}, R_{1}<R_{2}$, and $C_{1}<C_{2}$, two possible cases regarding $R_{1}-C_{1}<R_{2}-C_{2}$ and $R_{1}-C_{1}>R_{2}-C_{2}$ occur in user participation system. To simulate these two situations and compare with them, parameter setting is presented in Table 7.
Figure 7 concludes two cases mentioned in situation 5 . From Figure 7, some observations are concluded:

(a) Due to the unreasonable returns of both active and passive users (e.g., $R_{1}<C_{1}, R_{2}<C_{2}$ ), users prefer to choose passive strategy $(\bar{P}<0.5)$ without considering rewards (no matter $R_{1}-C_{1}<R_{2}-C_{2}$ or $\left.R_{1}-C_{1}>R_{2}-C_{2}\right)$

(b) From Figure 7, the probability for choosing active strategy (from 0.483 to 0.597 ) and average returns (from -2.4 to 3.8 ) continuously increase as the growth of rewards. This situation particularly occurs in the early stage of community's operation. As a new cocreation community opens, since there are no existing resources (e.g., knowledges and experiences) for users to exchange, both active and passive users cannot obtain enough returns. Thus, managers may set some rewards to attract more persons. For example, Pollen Club rewards their electronic equipment (e.g., phones and watch) for active users. Similar actions are also implemented by Xiaomi. These actions bring opportunities for creating a thriving community in the early stage of community's operation.

4.2. Computational Results Discussed from External Motivations. As Section 1 presents, the decision of user participation is also influenced by external motivations (e.g., number of users and group preference). These motivations can be treated as characteristics of user interactive network (e.g., network size and network structure). This section is to test the influence of external motivations on user participation behavior via analysis of user interactive network in NEGM. Specially, next sections, respectively, examine the effect of information noise in decision process $(\delta)$, network size $(N)$, number of small groups $(M)$, and size of small group (Group_Size). Further, four types of network (e.g., regular network, small-world network, scale-free network, and network with small groups) are employed to test the influence of network structure on the decision of user participation.

4.2.1. The Effect of Information Noise in Decision Process ( $\delta)$. Certain external events may fascinate the community and trigger off unexpected bursts of participation [20]. Here, information noise $(\delta)$ is set to test the effect of unexpected bursts on user participation. As Section 4.1 analyses, two main cases including $R_{1}-C_{1}<R_{2}-C_{2}$ and $R_{1}-C_{1}>R_{2}-$ $C_{2}$ exist in the user participation system. Thus, parameters of evolutionary game model are set based on these two cases. Besides, to exclude the effect of user interactive network, the related parameters are denoted as the same value. Bases on the characteristic of information noise mentioned in Section 3.2.3, the value of information noise increases 10 times at each change. Table 8 shows parameter setting.

Figure 8 concludes two cases mentioned in Table 8 .

From Figure 8, some observations are concluded:

(a) Information noise has a great influence on the decision of user participation. To further verify the 


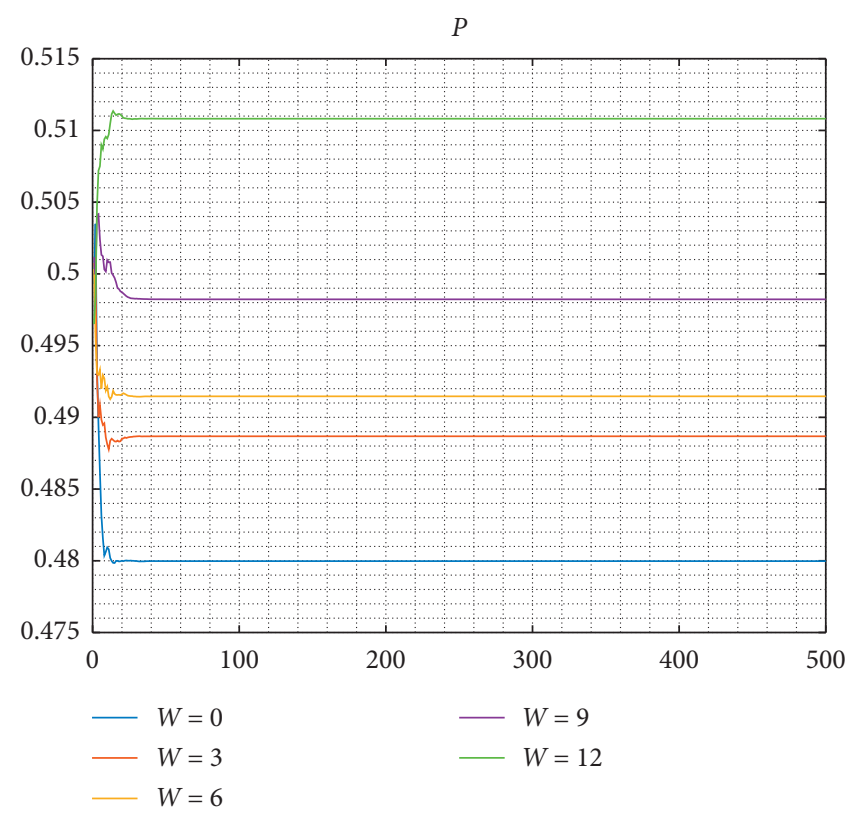

(a)

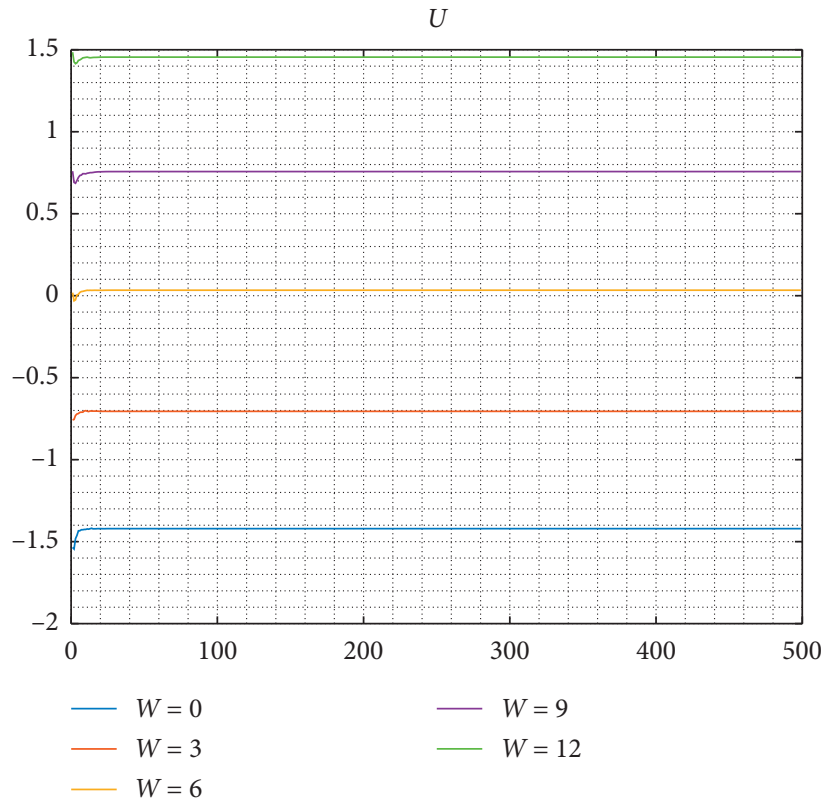

(b)
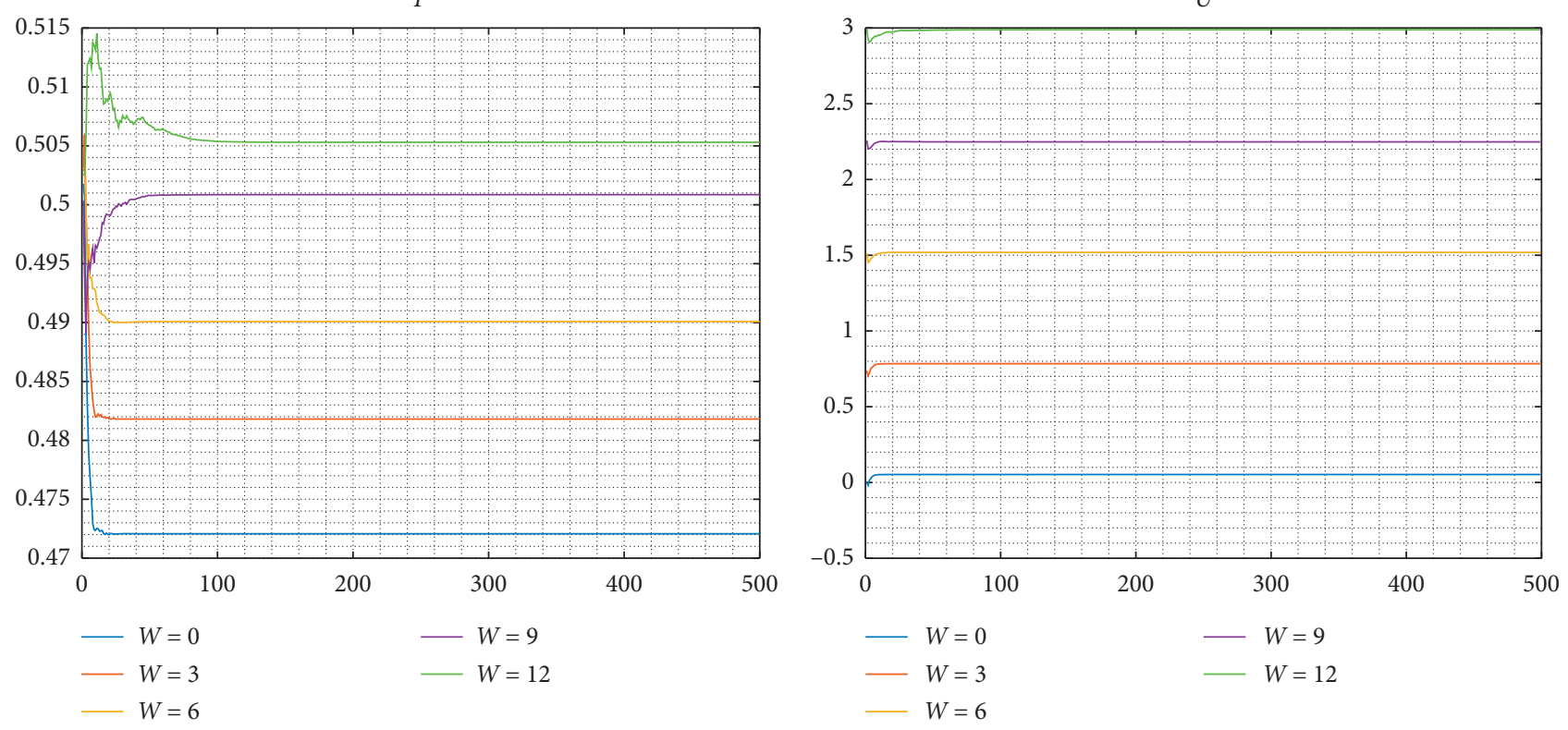

(c)

(d)

Figure 6: Continued. 
P

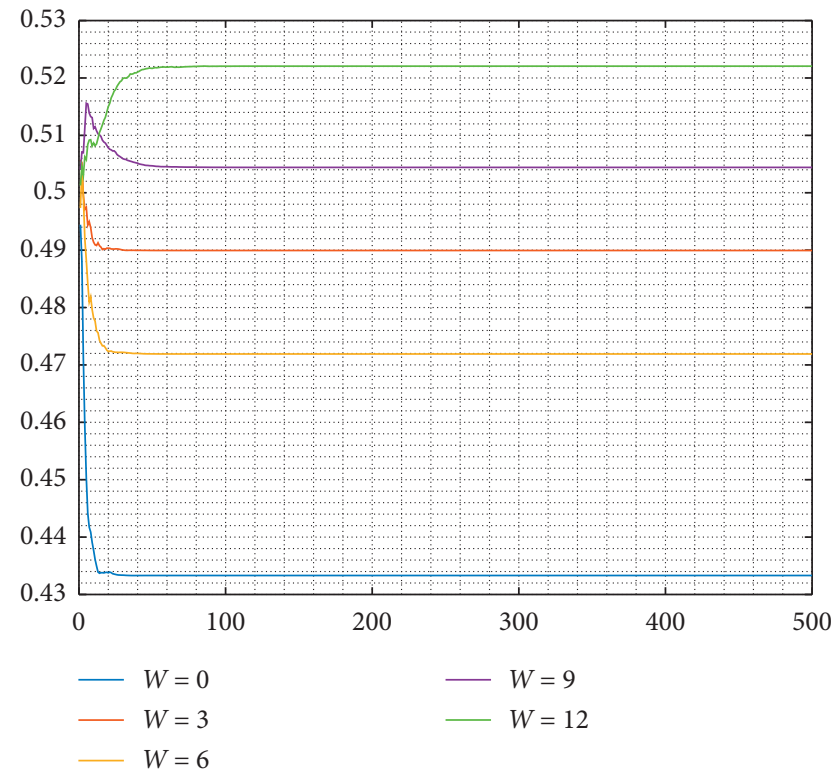

(e)

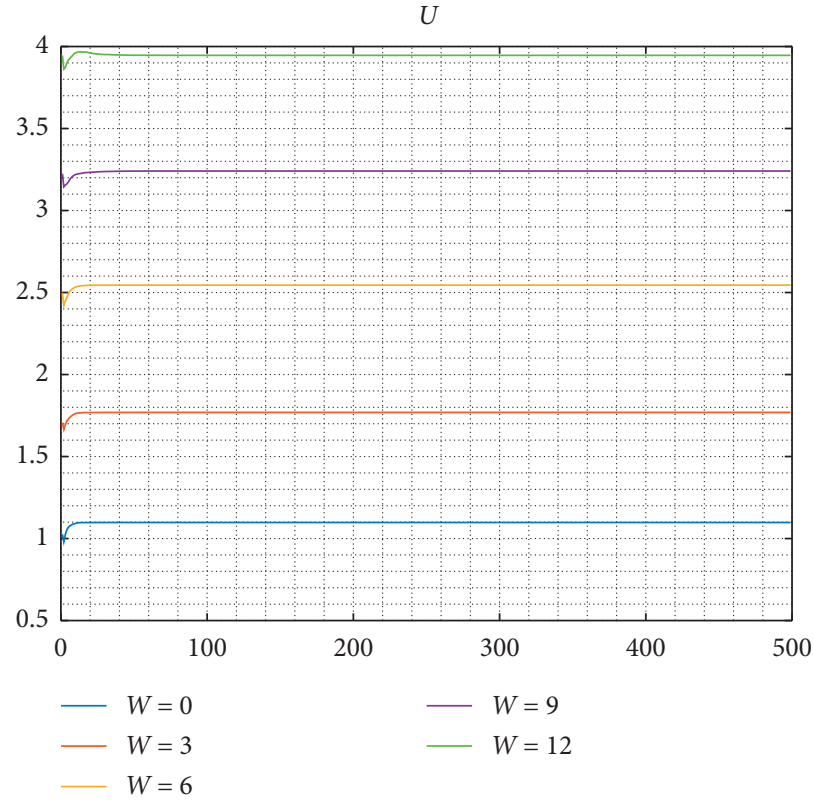

(f)

Figure 6: The simulated results of situation 4. (a) $\bar{P}$ (Case 1). (b) $\bar{U}$ (Case 1). (c) $\bar{P}$ (Case 2). (d) $\bar{U}$ (Case 2). (e) $\bar{P}($ Case 3 ). (f) $\bar{U}($ Case 3 ).

TABLE 7: Parameter setting of situation 5.

\begin{tabular}{lccccr}
\hline & $R_{1}$ & $R_{2}$ & $C_{1}$ & $C_{2}$ & $W$ \\
\hline Case 1: $R_{1}-C_{1}<R_{2}-C_{2}$ & 12 & 16 & 16 & 18 & {$[0,5,10,15,20]$} \\
Case 2: $R_{1}-C_{1}>R_{2}-C_{2}$ & 12 & 16 & 14 & 20 & {$[0,5,10,15,20]$} \\
\hline
\end{tabular}

significant influence, ANOVA analysis is employed to test the differences in means and variances at a $95 \%$ confidence level. When $p-$ value $<0.05$, it indicates that there is a critical difference between the test populations. All values (both Case 1 and 2) show significant differences.

(b) From Figure $8(\mathrm{a})$, when $\delta=0.1$, it indicates that users are completely rational, and they are sure to imitate their neighbors' strategies. In this case, users who choose active strategy obtain good returns from community. Thus, users will imitate their strategy and choose active participation with a probability of 0.9. However, strategy selection of active participation is inhibited by the growth of information noise. For example, when $\delta=100$ or $\delta=1000$, users show the bounded rationality, and they imitate their neighbors' strategies based on their character and mood. Thus, the probabilities for choosing active strategy, respectively, decline as 0.63 and 0.49 .

(c) Compared with results discovered in Figure 8(a), the completely reverse result that information noise promotes active participation is found. By reading the study of [39], the same result was also found in their study regarding human cooperation in networks. They explained that the proper information noise was helpful for cooperation promotion, especially in the context with many defectors.

4.2.2. The Effect of Network Size (N). According to empirical study created by [13], the number of users positively affects the intention of active participation in online communities. In this section, the number of users is treated as the network size to test the influence of number of users on the decision of user participation. Four types of network size (e.g., $N=[400,800,1200,1600])$ are considered here. Besides, to reduce the effect of information noise, $\delta$ is set as 0.1 . Other parameters are set as the same values which are detailed in Table 8 . The related results are presented in Table 9.

From Table 9, $p p$-value of Case 1Case $1(\leq 0.001)$ and Case $2(\leq 0.001)$ indicates that there is a significant difference among different network sizes. In other words, network size has a critical role on the decision of user participation. This result also verifies the hypothesis portrayed by [13].

4.2.3. The Effect of $M$ and Group_Size. As [35] argued, all online communities (including co-creation community) had their own special structures. For example, number of small groups is different. Pollen Club is divided as seven groups including experience sharing, suggestion for product improvement, feedback on product using, official activities, 


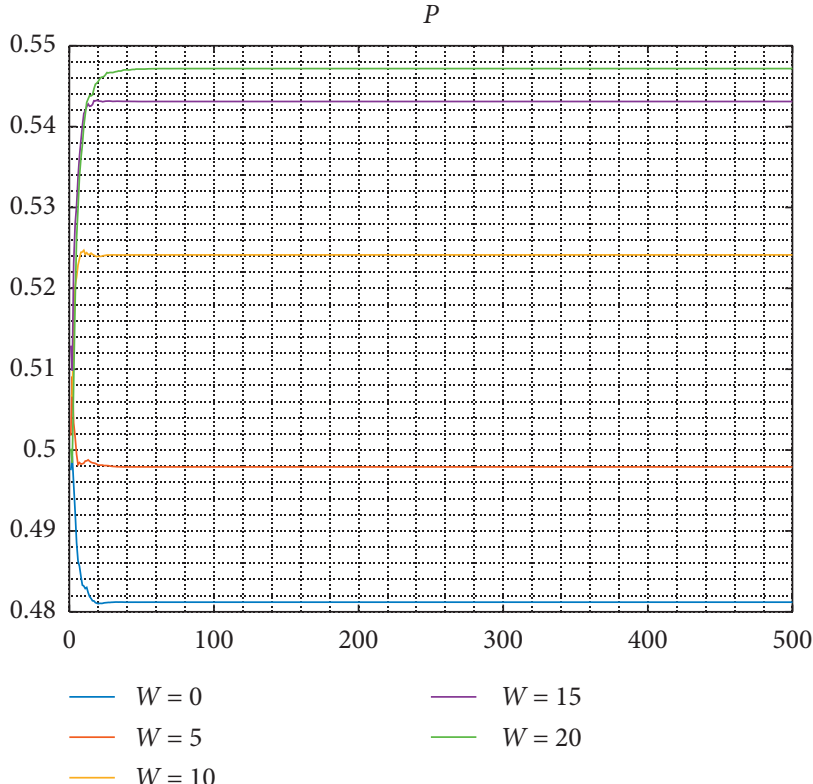

(a)

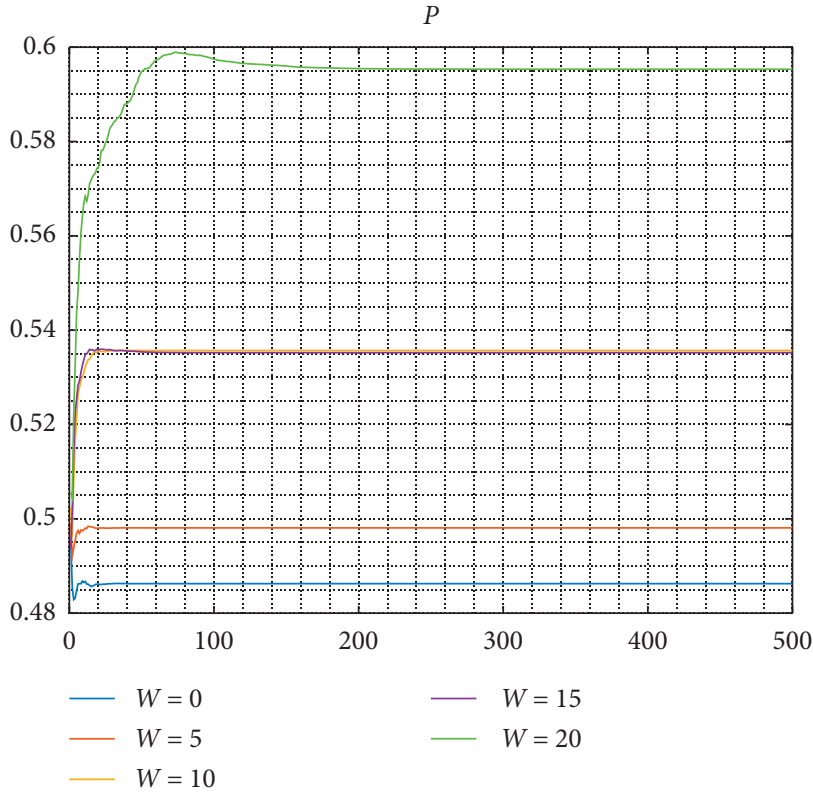

(c)

Figure 7: The simulated results of situation 5. (a) $\bar{P}$ (Case 1). (b) $\bar{U}$ (Case 1). (c) $\bar{P}$ (Case 2). (d) $\bar{U}$ (Case 2).

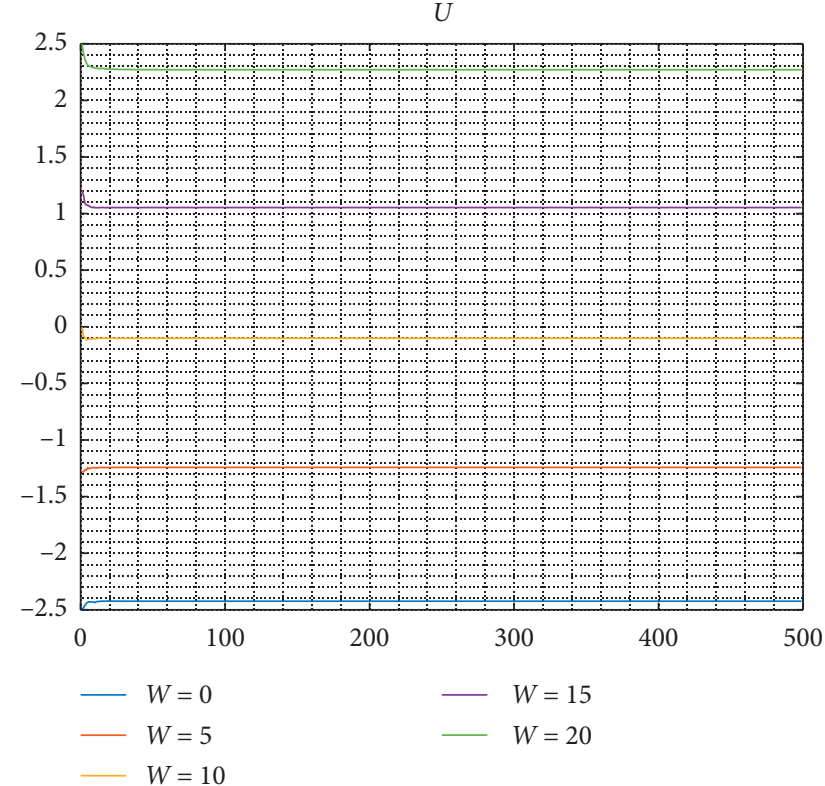

(b)

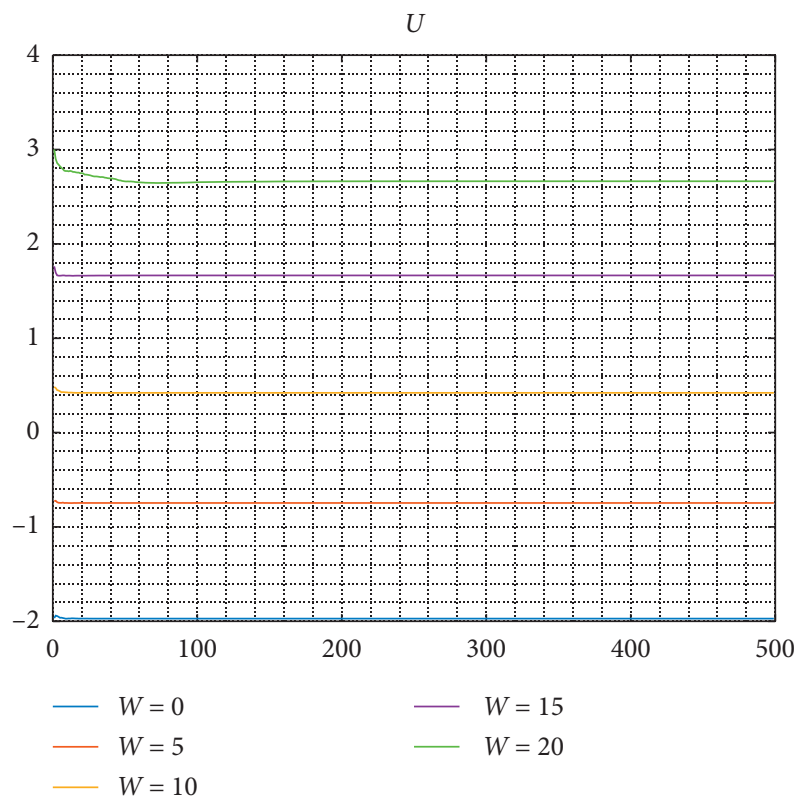

(d) product evaluation, resources for better using, and others. Xiaomi Club only has 5 groups (e.g., the best posts, photo sharing, proposal for activities, suggestion for product improvement, and asking for help) in their community. Thus, it is necessary to test the influence of special structures on user participation. According to user interactive network proposed in this paper, number of small groups and size of small groups are considered.

Refer to size of small groups; two types of networks (e.g., uniform network and non-uniform network) should be introduced here. Main differences between them are presented in Figure 9. Specially, Figure 9(a) is a uniform network which 10 nodes, respectively, exist in each small group. Figure 9(b) is a non-uniform network which different number of nodes (e.g., 5, 10, and 15), respectively, exist in each small group. Motivated by the result observed in Section 4.2.2, this section assumes that size of small groups also has a great impact on decision-making.

To test the influence of $M$ and Group_Size on the decision of user participation, three types of computational experiments are designed. Specially, experiment 1 and experiment 2 aim to analyze the influence of $M$ on user participation from perspectives of uniform and nonuniform networks. Experiment 3 aims to make a comparison between 
TABle 8: Parameter setting considering the change of $\delta$

\begin{tabular}{|c|c|c|c|c|c|c|c|c|}
\hline & $R_{1}$ & $R_{2}$ & $C_{1}$ & $\mathrm{C}_{2}$ & $W$ & $N$ & $T$ & $\delta$ \\
\hline Case 1: $R_{1}-C_{1}>R_{2}-C_{2}$ & 18 & 6 & 10 & 8 & 0 & 2000 & 500 & {$[0.1,1,10,100,1000]$} \\
\hline Case 2: $R_{1}-C_{1}<R_{2}-C_{2}$ & 18 & 16 & 22 & 14 & 0 & 2000 & 500 & {$[0.1,1,10,100,1000]$} \\
\hline
\end{tabular}

P

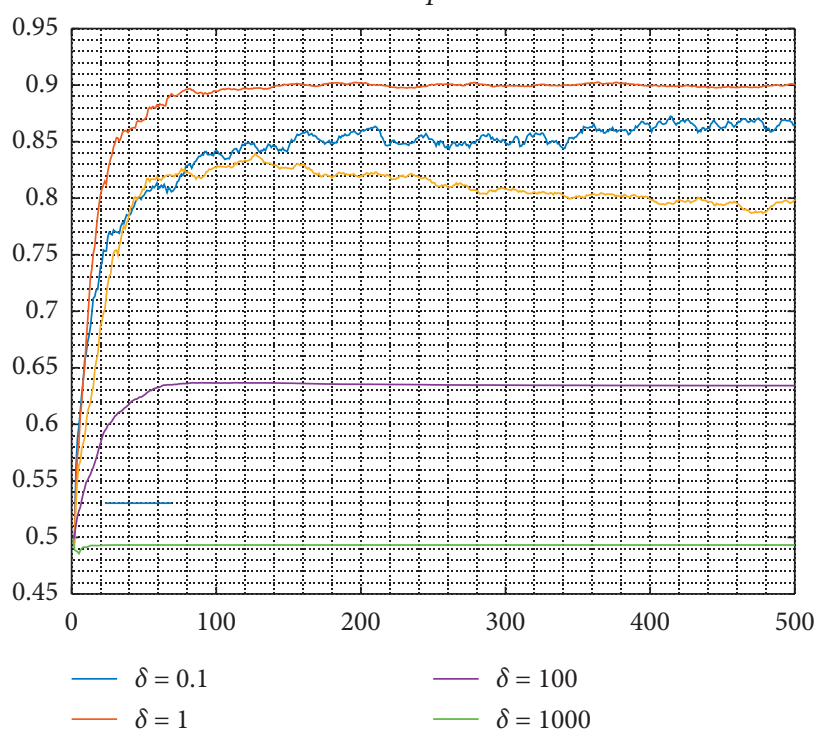

(a)

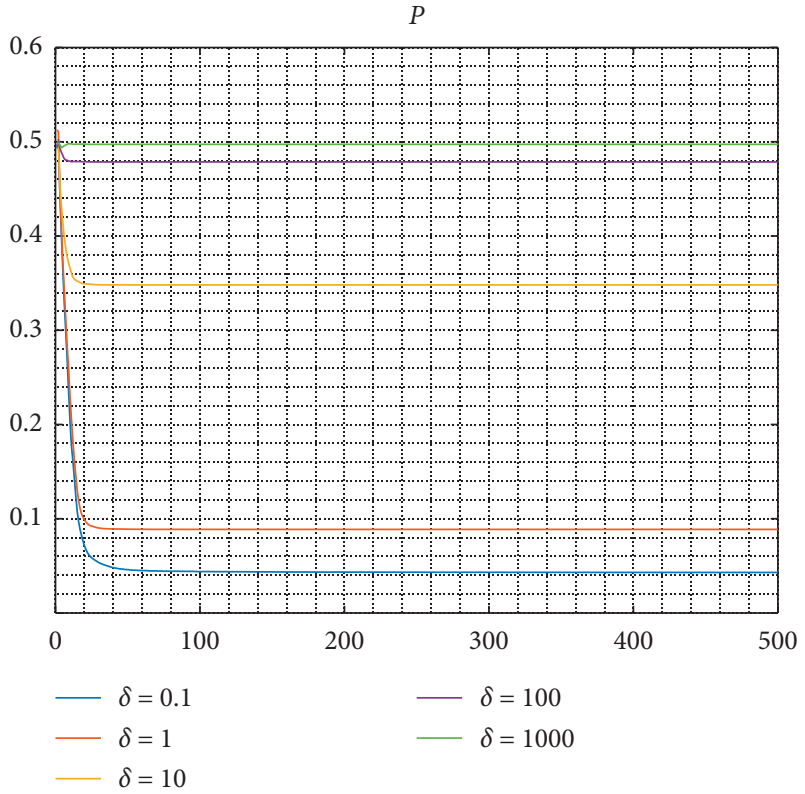

(b)

FIgURE 8: The simulated result considering the change of information noise. (a) $\bar{P}($ Case 1). (b) $\bar{P}$ (Case 2).

TABLE 9: Summary of simulated result considering the change of network size.

\begin{tabular}{|c|c|c|c|c|c|}
\hline & Network size & Convergence result of $\bar{P}$ & $p$-value of ANOVA & Homogeneity tests & $p$-value regarding nonparametric tests \\
\hline \multirow{3}{*}{ Case 1} & $N=400$ & 0.849 & \multirow{3}{*}{$\leq 0.001$} & \multirow{3}{*}{$\leq 0.001$} & \multirow{3}{*}{$\leq 0.001$} \\
\hline & $N=1200$ & 0.870 & & & \\
\hline & $N=1600$ & 0.901 & & & \\
\hline \multirow{4}{*}{ Case 2} & $N=400$ & 0.046 & \multirow{4}{*}{$\leq 0.001$} & \multirow{4}{*}{0.945} & \multirow{4}{*}{-} \\
\hline & $N=800$ & 0.041 & & & \\
\hline & $N=1200$ & 0.027 & & & \\
\hline & $N=1600$ & 0.044 & & & \\
\hline
\end{tabular}

* Case 1 and Case 2, respectively, indicates that $R_{1}-C_{1}>R_{2}-C_{2}$ and $R_{1}-C_{1}<R_{2}-C_{2}$, which is detailed in Table 8 .

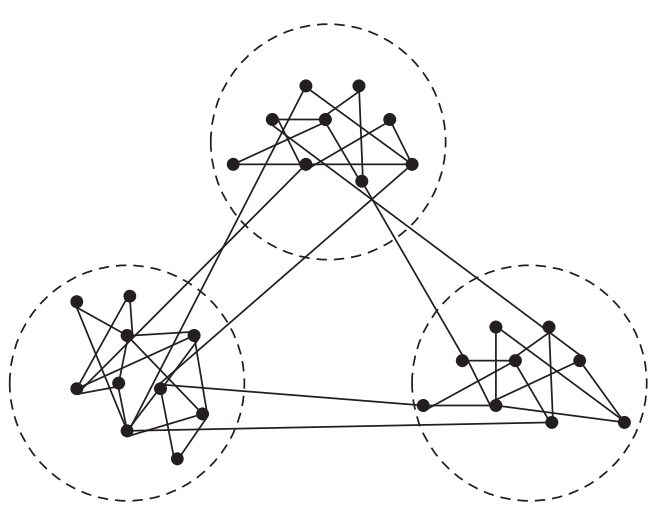

(a)

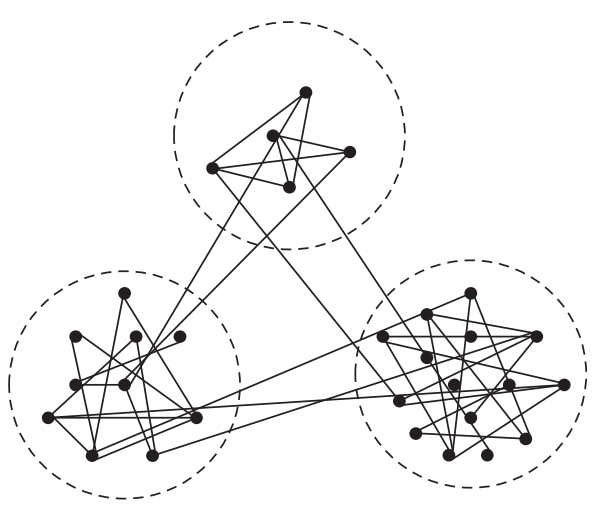

(b)

FIGURE 9: Two types of networks regarding uniform network and nonuniform network, (a) uniform network, (b) nonuniform network. 
uniform and nonuniform networks. To exclude the impact of other parameters, all experiments of other parameters are denoted as $R_{1}=18, R_{2}=6, C_{1}=10, C_{2}=8, W=0, \delta=0.1$, $N=2000$ and $t=500$. Table 10 shows parameter setting of $M$ and Group_Size. Specially, when $M=3$, Group_Size of uniform network is set as 666, 666, and 668. Besides, Group_Size of non-uniform network is denoted as 50, 50, and 1900.

Figure 10 concludes all computational results mentioned in three experiments.

From Figure 10, some observations are concluded:

(a) Figures 10(a)-10(d) shows that significant different exists in the nonuniform network but not in the uniform network. The same result is also found by ANOVA analysis of their values. This phenomenon can be explained by the non-uniform distribution of users. When the biggest small group obtains good returns via choosing active strategy, the successful strategy will spread in populations. Otherwise, the defection strategy (e.g., passive strategy) is popular. In other words, group selection depends on the successful strategy adopted by the biggest small group.

(b) From Figures 10(e) and 10(f), uniform network shows a better performance on promotion of active participation. The same result also can be discovered from comparison between Figures 10(a) and 10(c). This result can be illustrated by the study of [40]. They argued that it caused by the detailed evaluation function of the players' success and the different evolutionary rules.

4.2.4. The Effect of Different Network Structures. Although empirical study discussed in Section 3.2.1 presents that user interactive network of co-creation community has scale-free features and the occurrence of small groups, other studies on user interactive network of online communities have identified that other types of networks (e.g., regular network, small-world network, and purely scale-free network) can also be employed to describe user interaction in online communities. Thus, this section aims to analyze the effect of different network structures on the decision of user participation.

As Section 4.2.1 presents, two types of cases regarding $R_{1}-C_{1}<R_{2}-C_{2}$ and $R_{1}-C_{1}>R_{2}-C_{2}$ exist in the user participation system. Thus, parameters of evolutionary game model are set as the same value detailed in Table 8 . Besides, to exclude the effect of network size and information noise, the fixed value of $N=2000$ and $\delta=0.1$ is denoted here. The variable of this section is network structure which is generated by different network generation models [41]. Figure 11 shows the tendency of evolutionary strategies and average returns.

From Figure 11, some observations are concluded:

(a) Compared with regular network and small-world network, purely scale-free network and network with small groups inhibit active participation in Figure 11(a). Similar result was also found in the study of human cooperation [38]. Wu et al. [38] explained that long-tail degree distribution was the main reason. This result indicates that uniform distribution (e.g., regular network and small-world network) is more suitable for promoting active participation, which enhances the result found in Section 4.2.3. From Figure 11(b), users in network with small groups obtain the smallest average returns $(\bar{U}<1)$ from communities. However, the probability for choosing active strategy $(\bar{P}=0.78)$ is not obviously smaller than the probability in other network structures.

(b) There are no significant differences among lines depicted in Figure 11(c). It indicates that network structure has a limited role under the circumstance of $R_{1}-C_{1}<R_{2}-C_{2}$.

4.2.5. Discussions and Managerial Insights. Based on computational experiments, some discussions and managerial insights are summarized as follows:

(a) Section 4.1 details all possible situations in real practice. The decision of user participation is significantly influenced by return-cost analysis based on the evaluation of several internal motivations. The probability for choosing active strategy depends on return allocation for active and passive users. Managers should carefully focus on return allocation for active users and ensure that returns obtained by active users are more substantial than those gained by passive users. In other words, the rule of "more pay for more work" is necessary in the user participation system. Similar result was also suggested by [17].

Experimental results indicate that rewards effectively promote active participation in many situations. However, the probability for choosing active participation does not continuously increase with the growth of rewards, which results from the special structure of interactive network (e.g., scale-free feature and the occurrence of small groups). For example, users are generally divided into several parts based on different small groups. The decisions of them are mainly affected by the strategy which is widely adopted by their own small groups. If a passive strategy is initially adopted by populations, it is hard to change their initial strategy via rewards.

Therefore, the influence of rewards on the promotion of active participation does not make sense on every occasion. Managers should design specific rewards based on different situations. For example, in the early stage of the community's operation, rewards should be appropriated among both active and passive users. By contrast, rewards are unnecessary in the recession of the community's operation, especially when the budget for the community's maintenance is not sufficient.

(b) Information noise has a great influence on user participation. Specially, when active participation is 
Table 10: Parameter setting of $M$ and Group_Size

\begin{tabular}{lrr}
\hline & $M$ & Group_Size \\
\hline Experiment 1: uniform network & {$[666,666,668] ;$} & {$[500,500,500,500] ;$} \\
& {$[3,4,5]$} & {$[400,400,400,400,400]$.} \\
Experiment 2: nonuniform network & {$[50,50,1900] ;$} & {$[50,50,50,1850] ;$} \\
Experiment 3: comparison between uniform network and nonuniform networks & {$[3,4,5]$} & {$[50,50,50,50,1800]$.} \\
& {$[500,500,500,500] ;$} \\
& {$[50,50,50,1850]$.} \\
\hline
\end{tabular}

$P$

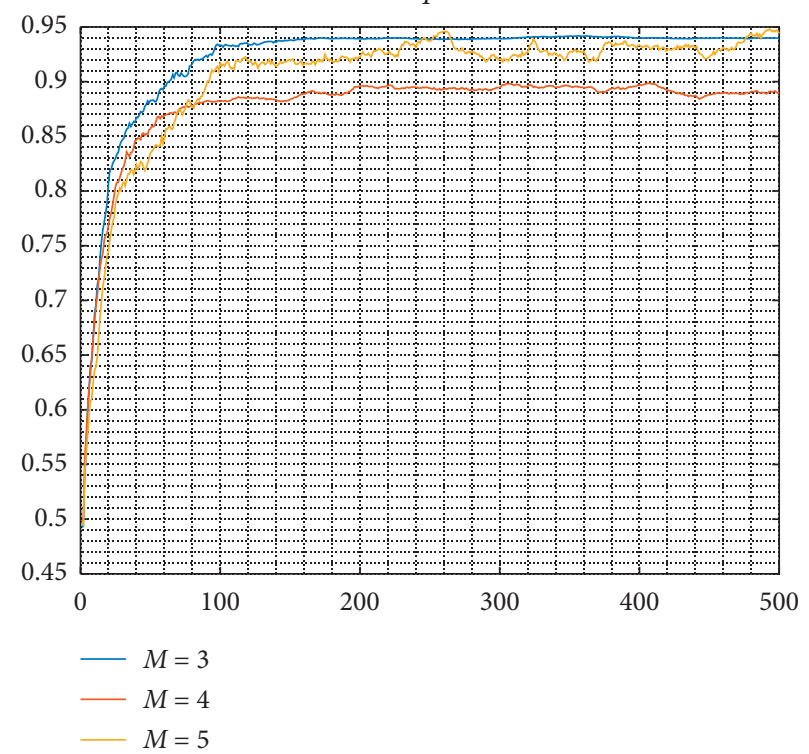

(a)

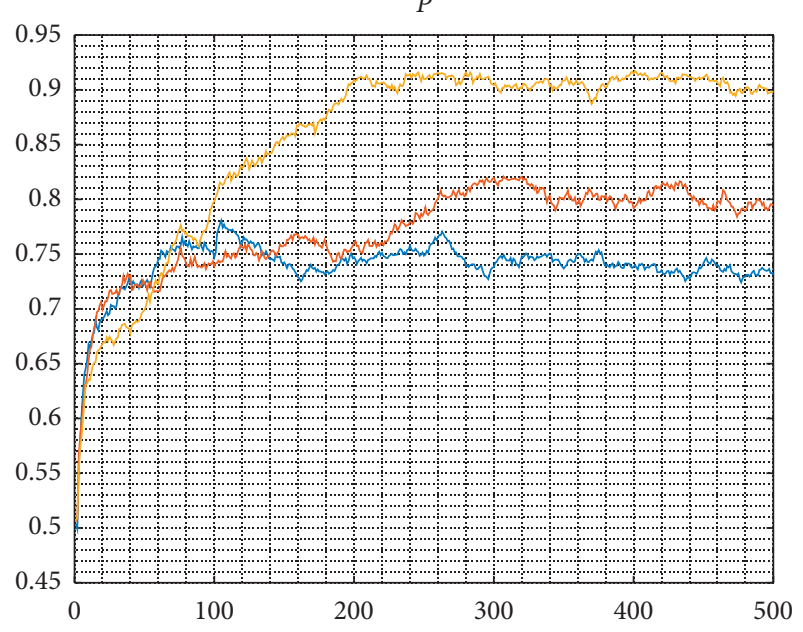

$-M=3$

- $M=4$

$M=5$

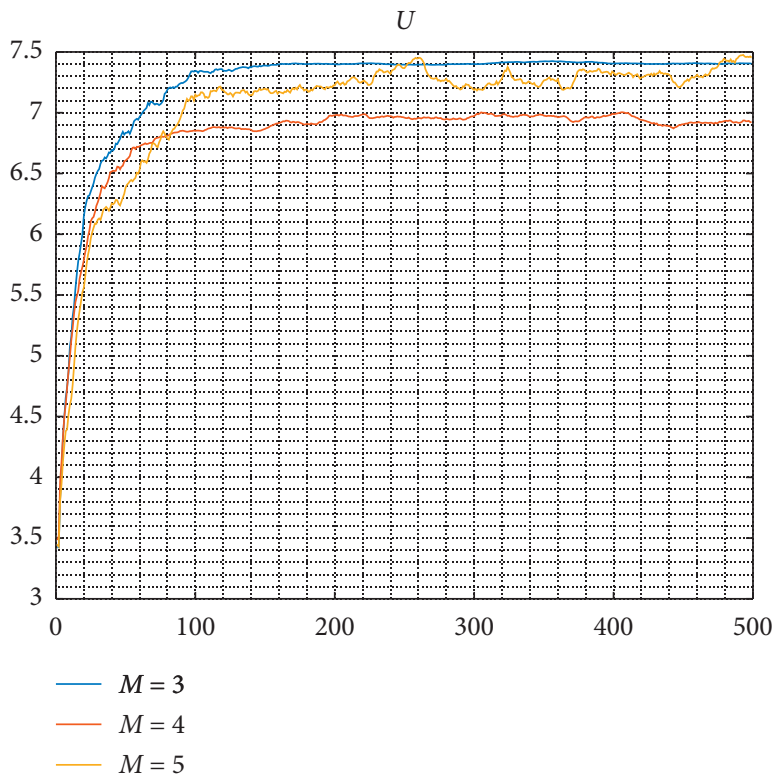

(b)

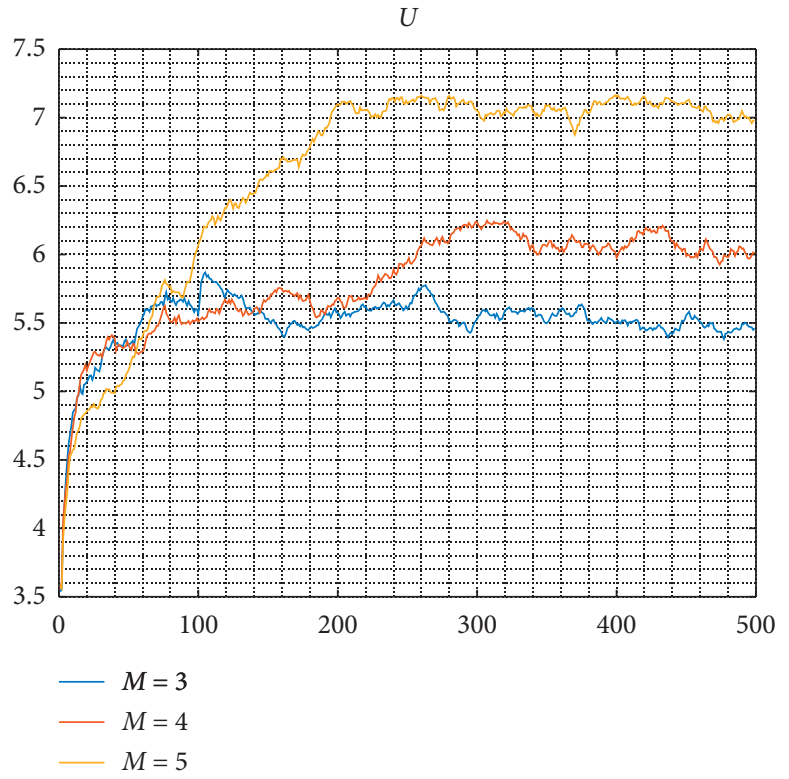

(d)

(c)

FIgURE 10: Continued. 


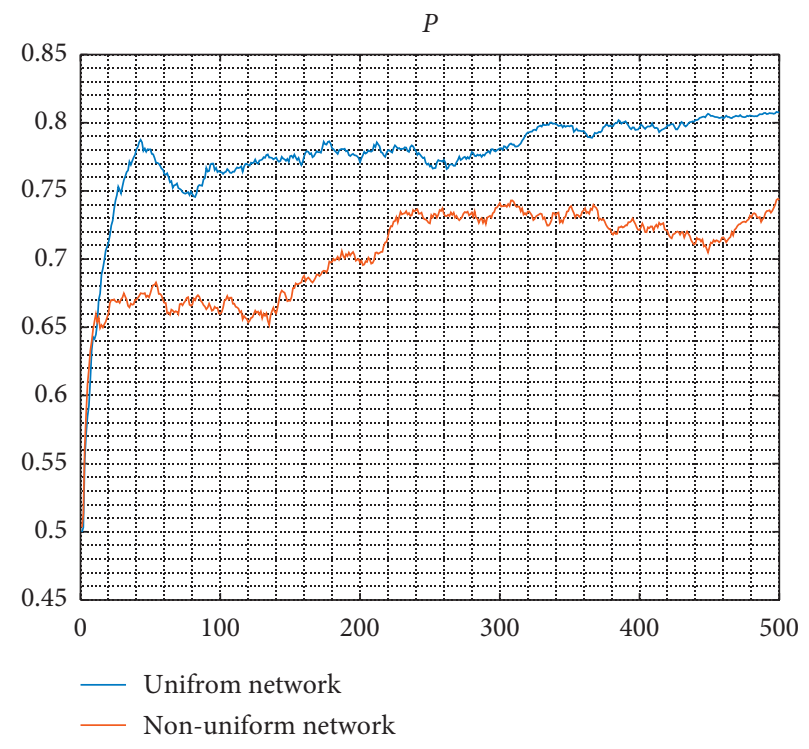

(e)

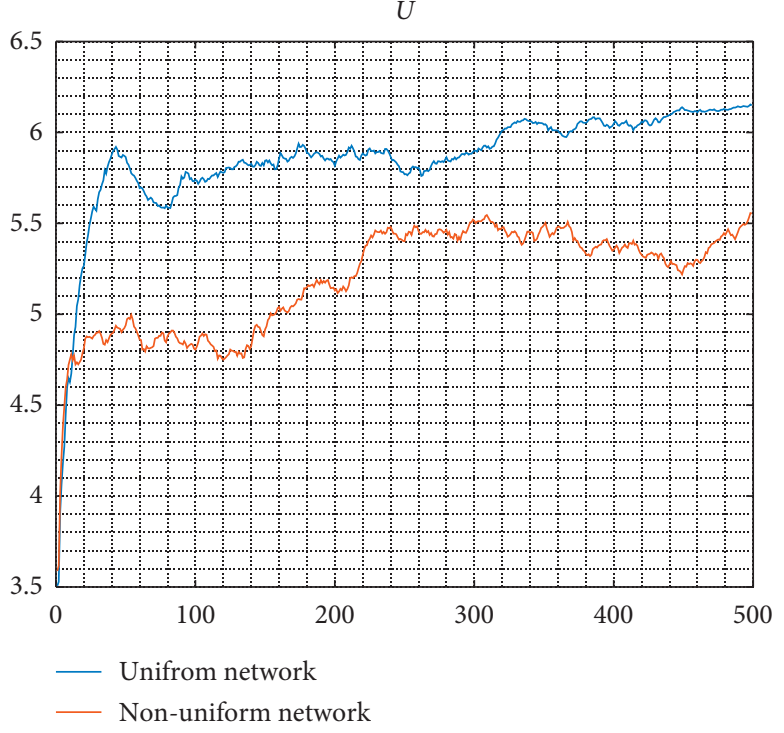

(f)

Figure 10: The simulated result considering the change of $M$ and Group_Size. (a) $\bar{P}$ (Experiment 1). (b) $\bar{U}$ (Experiment 1$)$. (c) $\bar{P}($ Experiment 2). (d) $\bar{U}$ (Experiment 2). (e) $\bar{P}$ (Experiment 3). (f) $\bar{U}$ (Experiment 3 ).

the dominant strategy in populations, the falling tendency of active participation occurs in decision system with the growth of information noise. By contrast, when a passive strategy is adopted by many users, the increasing tendency of active participation occurs with the growth of information noise.

According to equation (16), information noise closely associates with the characteristic of users. The small value of information noise indicates that users are completely rational, while the big value of information noise indicates that users show the bounded rationality. When passive participation is a dominant strategy in the early stage of the community's operation, managers should make some attempts (e.g., employing some persons to share experience and knowledge in a community, and organizing some activities to attract more users) to promote active participation via enhancing information noise. Influenced by these information noises, users showing bounded relationality will make decisions based on their moods and characteristics. Thus, the dominant strategy of passive participation will be changed next time.

(c) Network size plays a critical role in promoting active participation. This result is also found in the empirical study of [13]. According to their investigation, lurkers' decisions are significantly influenced by network size. Lurkers prefer to share experience and knowledge in a thriving community. At first, they prefer to respond to posts rather than to publish posts. In this context, mangers can create some hot topics and recommend these topics for lurkers. This action will attract more lurkers to discuss their ideas in communities. Besides, it is also necessary to expand user base in a short time, which is also suggested in [5].

(d) Due to the non-uniform degree distribution of user interactive network, active participation is significantly promoted with the growing number of small groups. Besides, uniform network performs well on the promotion of active participation, compared with the result of nonuniform network. In a nonuniform network, the dominant strategy is easily controlled by small groups with many participants. The decisions of other users are significantly affected by their decisions. Thus, it is necessary to detect the number of users in each small group and maintain similar users in each group. If the user interactive network shows nonuniform characteristic, it is necessary to promote active participation via other actions (e.g., rewards setting, enhancing information noise, and expanding user base).

(e) Compared with the regular network and small-world network, purely scale-free network and network with small groups inhibit active participation when the dominant strategy is active participation. Almost 80 percent of online communicating networks have scale-free features, and these characteristics are not conductive to the promotion of active participation. Fortunately, the small-world feature (e.g., the clustering coefficient is high, and the average shortest path is short) is also identified in many real networks, which can change the current situation from passive participation to active participation. Online communications usually follow the principle of the "three degrees of influence rule." It indicates that two strangers can make the interaction via three 


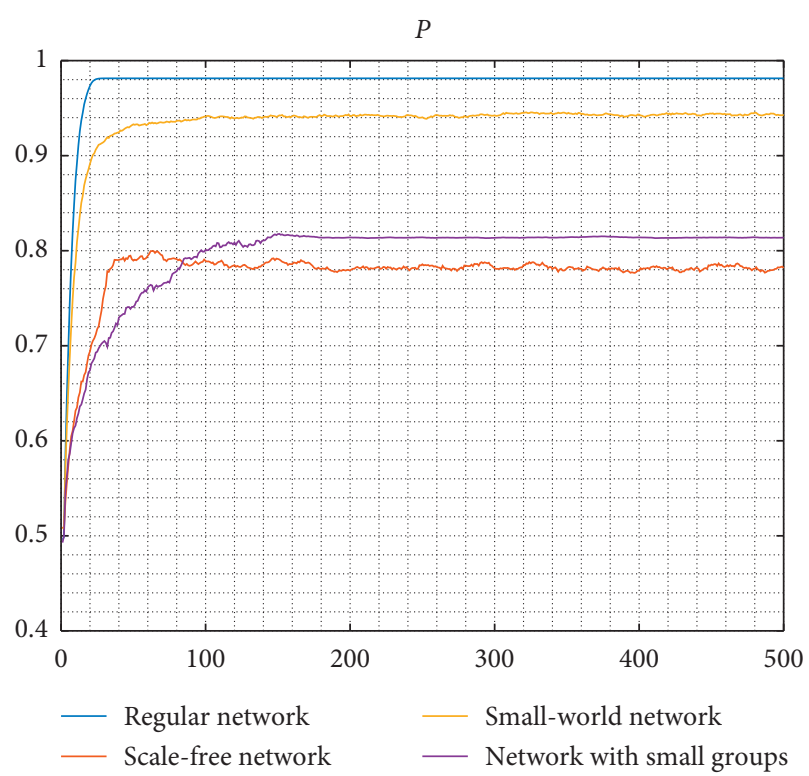

(a)

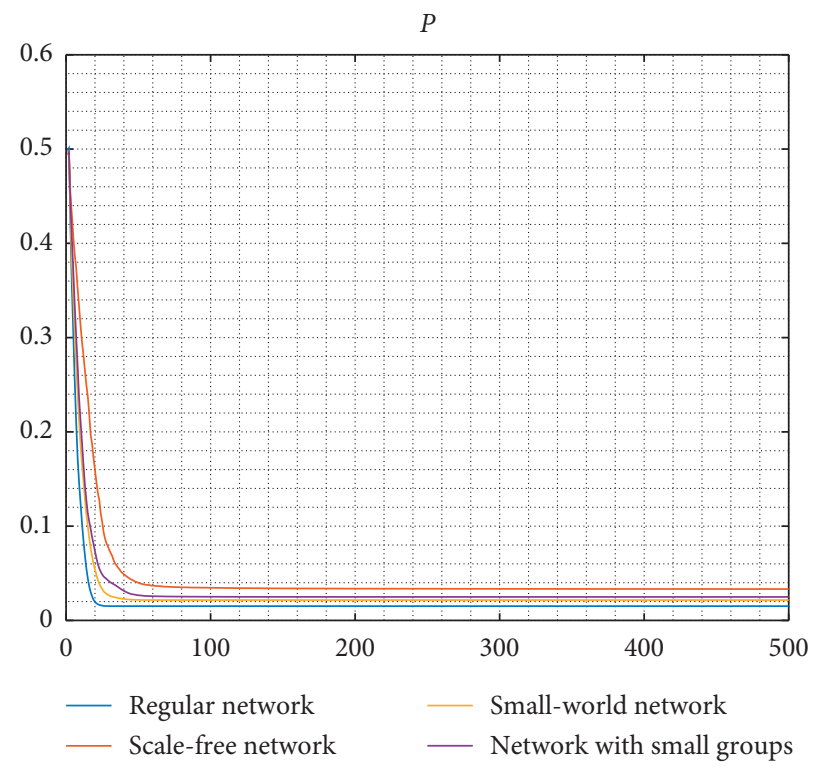

(c)

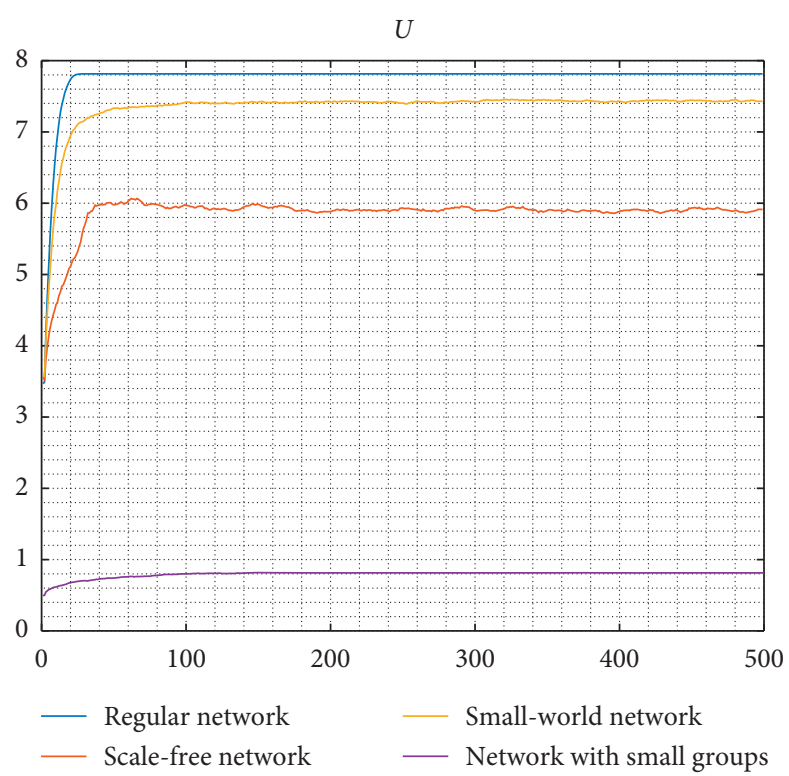

(b)

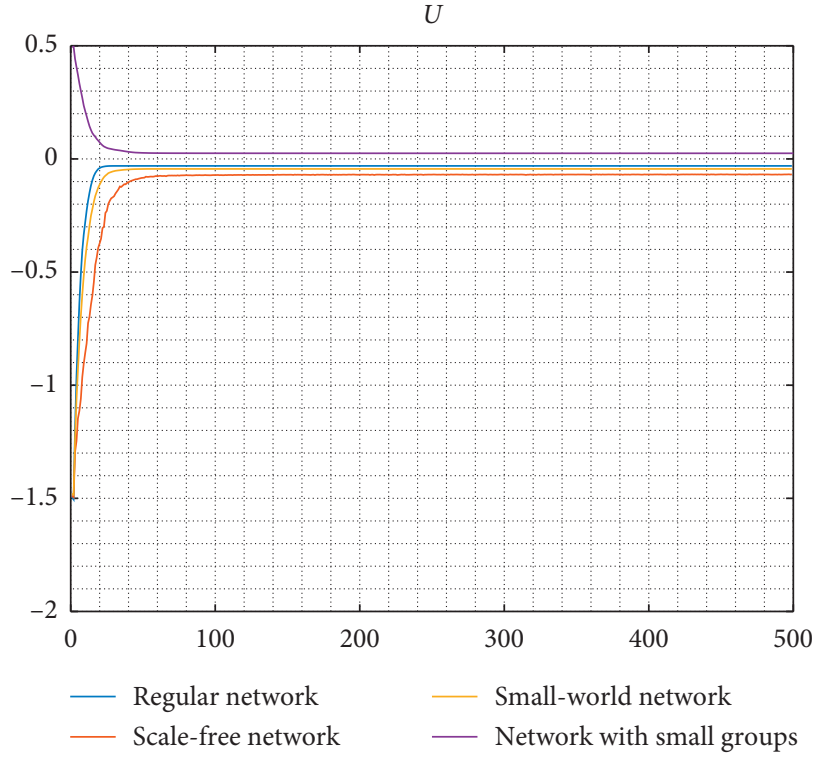

(d)

Figure 11: The simulated result considering the change of $M$ and Group_Size. (a) $\bar{P}($ Case 1). (b) $\bar{U}$ (Case 1). (c) $\bar{P}($ Case 2). (d) $\bar{U}($ Case 2).

relationships. Based on this finding, actions (e.g., publishing some hot-topic posts to attract more discussions, looking for the lead users to closely connect many persons, and organizing group-based competitions) should be taken to shorten the path among several users.

\section{Conclusions}

In this paper, the mechanism regarding how internal and external motivations influence the decision of users in participation in co-creation community is analyzed by using NEGM. User behavior (e.g., active participation and passive participation) and the evolutionary process of strategy selection are examined via computational experiments. Two main findings are concluded. (a) User participation behavior is significantly influenced by return-cost analysis based on the evaluation of internal motivations. Rewards sometimes enhance and sometimes inhibit user active participation. (b) User participation behavior is closely associated with network size, network structure, and information noise in the decision-making process.

This paper employs NEGM and computational experiments to investigate the evolution of user participation behavior in co-creation communities. It contributes to the recent literature in threefold manner. (a) Different from extensive studies focusing on identifying motivation of user participation, this paper concentrates on the motive of continuous user 
participation behavior and the mechanism regarding whether users should actively participate or not, when to actively participate and how such a strategy can perform well. It fills up the research gap argued by [18]. (b) The influence of external motivations (e.g., network size, network structure, and information noise) on user participation behavior is analyzed by using NEGM, which transforms the research focus from individual-level to group-level effects [16]. (c) The computational experiments proposed in this paper are helpful for visualizing the evolution of users' strategies, grasping the dynamic evolution features in user participation games over time, and exploring the change of strategies in different situations.

This paper analyses the mechanism regarding how different motivations affect the decision of user participation. The practical recommendations for the sustainable operation of cocreation community are summarized. Firstly, for individual users, some proposed strategy should be taken, such as maximizing the returns of active participates, minimizing the returns of passive users via free-riding behavior, and motivating passive users to actively participate in order to maximize the group returns rather than eliminating them from communities. Secondly, if the strategies mentioned onwards are to be enticed to participates, external motivations, such as information noise, network size, and network structure, should be taken into consideration. Thirdly, rewards do not make sense all the time, which differs from recent studies on incentive design. Managers should implement the specific policy to promote active participation.

Limitations and future directions are concluded as follows. Firstly, although external motivations (e.g., network structure and information noise) are identified as critical factors influencing the decision of user participation, the question regarding how to manage user interactive network in a proper approach is not clear. The valuable direction for future research is suggested to explore the reconfiguration of user interactive network [42]. Secondly, all data used in computational experiments are hypothetical. Real data (particularly, how to abstract real data from practices is a difficult work) are recommended for the further research, although hypothetical data are also suitable for describing all possible situations in real practices. Thirdly, in this paper, all users are assumed as homogeneous stakeholders in a cocreation community. It is suggested that the characteristics of different users should be further distinguished in the future, so as to develop an asymmetrical evolutionary game model for describing their returns and costs in the user participation system, and thus making a comparison with the results observed in this paper. Fourthly, the improved NEGM should be developed to fit the real practice. For example, a memory of the actions and a genetic evolution (not just to imitate the strategy of the neighbor with a better reward) is recommended for future research The trade-off among different users can be optimized by the multiobjective programming model [43]..

\section{Data Availability}

The data used to support the finding of this paper are included within this paper.

\section{Conflicts of Interest}

The authors declare no conflicts of interest.

\section{Acknowledgments}

This work was supported by National Natural Science Foundation of China (Grant nos. 71672074, 72072072, and 71904021), Natural Science Foundation of Guangdong Province of China (No. 2019A1515010045), 2018 Guangzhou Leading Innovation Team Program (China) (Grant no. 201909010006) and Social Science Planning Project in Chongqing (Grant no. 2019QNGL27).

\section{References}

[1] H. J. Lee, D.-H. Lee, C. R. Taylor, and J.-H. Lee, "Do online brand communities help build and maintain relationships with consumers? A network theory approach," Journal of Brand Management, vol. 19, no. 3, pp. 213-227, 2011.

[2] Y. Yi and T. Gong, "Customer value co-creation behavior: scale development and validation," Journal of Business Research, vol. 66, no. 9, pp. 1279-1284, 2013.

[3] N. Ind, N. Coates, and K. Lerman, "The gift of co-creation: what motivates customers to participate," Journal of Brand Management, vol. 27, no. 2, pp. 181-194, 2020.

[4] S. Kamboj and Z. Rahman, "Understanding customer participation in online brand communities," Qualitative Market Research: An International Journal, vol. 20, no. 3, pp. 306-334, 2017.

[5] X. Lu, C. W. Phang, and J. Yu, "Encouraging participation in virtual communities through usability and sociability development," ACM SIGMIS Database: The DATABASE for Advances in Information Systems, vol. 42, no. 3, pp. 96-114, 2011.

[6] H.-T. Tsai, H.-C. Huang, and Y.-L. Chiu, "Brand community participation in Taiwan: examining the roles of individual-, group-, and relationship-level antecedents," Journal of Business Research, vol. 65, no. 5, pp. 676-684, 2012.

[7] J. Kang, L. Tang, and A. M. Fiore, "Enhancing consumerbrand relationships on restaurant Facebook fan pages: maximizing consumer benefits and increasing active participation," International Journal of Hospitality Management, vol. 36, pp. 145-155, 2014.

[8] B. Xu and D. Li, "An empirical study of the motivations for content contribution and community participation in Wikipedia," Information \& Management, vol. 52, no. 3, pp. 275-286, 2015.

[9] J. Nielsen, "Participation inequality: encouraging more users to contribute," 2006, https://www.researchgate.net/ publication/200772620_Participation_Inequality_ Encouraging_More_Users_to_Contribute.

[10] T. Zhou, "Understanding users' participation in online health communities: a social capital perspective," Information Development, vol. 36, no. 3, pp. 403-413, 2020.

[11] D. Roberts, M. Hughes, and K. Kertbo, 'Exploring consumers' motivations to engage in innovation through co-creation activities," European Journal of Marketing, vol. 48, no. 1-2, pp. 147-169, 2014.

[12] C. Chen, R. Du, J. Li, and W. Fan, "The impacts of knowledge sharing-based value co-creation on user continuance in online communities," Information Discovery and Delivery, vol. 45, no. 4, pp. 227-239, 2017. 
[13] C. Fang and J. Zhang, "Users' continued participation behavior in social Q\&A communities: a motivation perspective," Computers in Human Behavior, vol. 92, pp. 87-109, 2019.

[14] S. Malinen, "Understanding user participation in online communities: a systematic literature review of empirical studies," Computers in Human Behavior, vol. 46, pp. 228-238, 2015.

[15] K. Sigmund and M. A. Nowak, "Tides of tolerance," Nature, vol. 414, no. 6862, pp. 403-405, 2001.

[16] P. Shukla and J. Drennan, "Interactive effects of individualand group-level variables on virtual purchase behavior in online communities," Information \& Management, vol. 55, no. 5, pp. 598-607, 2018.

[17] A. Al-Dhanhani, R. Mizouni, H. Otrok, and A. Al-Rubaie, "A game theoretical model for collaborative groups in social applications," Expert Systems with Applications, vol. 41, no. 11, pp. 5056-5065, 2014.

[18] G. Jiang, F. Ma, J. Shang, and P. Y. K. Chau, "Evolution of knowledge sharing behavior in social commerce: an agentbased computational approach," Information Sciences, vol. 278, pp. 250-266, 2014.

[19] K. Musial, M. Budka, and K. Juszczyszyn, "Creation and growth of online social network," World Wide Web, vol. 16, no. 4, pp. 421-447, 2013.

[20] J. Vassileva, "Motivating participation in social computing applications: a user modeling perspective," User Modeling and User-Adapted Interaction, vol. 22, no. 1-2, pp. 177-201, 2012.

[21] T. Kohler, J. Fueller, D. Stieger, and K. Matzler, "Avatar-based innovation: consequences of the virtual co-creation experience," Computers in Human Behavior, vol. 27, no. 1, pp. 160-168, 2011.

[22] H. Akman, C. Plewa, and J. Conduit, "Co-creating value in online innovation communities," European Journal of Marketing, vol. 53, no. 6, pp. 1205-1233, 2019.

[23] H.-H. M. Lee and W. van Dolen, "Creative participation: collective sentiment in online co-creation communities," Information \& Management, vol. 52, no. 8, pp. 951-964, 2015.

[24] A. R. Lee and K. K. Kim, "Customer benefits and value cocreation activities in corporate social networking services," Behaviour \& Information Technology, vol. 37, no. 7, pp. 675-692, 2018.

[25] M. Chepurna and J. R. Criado, "Identification of barriers to co-create on-line: the perspectives of customers and companies," Journal of Research in Interactive Marketing, vol. 12, no. 4, pp. 452-471, 2018.

[26] H. Shen, L. Wu, S. Yi, and L. Xue, "The effect of online interaction and trust on consumers' value co-creation behavior in the online travel community," Journal of Travel \& Tourism Marketing, vol. 37, no. 4, pp. 418-428, 2020.

[27] T. Chen, J. Drennan, L. Andrews et al., "User experience sharing: understanding customer initiation of value co-creation in online communities," European Journal of Marketing, vol. 52, no. 5-6, pp. 1154-1184, 2018.

[28] C. Li, F. Zhang, C. Cao, Y. Liu, and T. Qu, "Organizational coordination in sustainable humanitarian supply chain: an evolutionary game approach," Journal of Cleaner Production, vol. 219, pp. 291-303, 2019.

[29] L. Xie and H. Han, "Capacity sharing and capacity investment of environment-friendly manufacturing: strategy selection and performance analysis," International Journal of Environmental Research and Public Health, vol. 17, no. 16, p. 5790, 2020.

[30] Z. H. Rong, X. R. Xu, and Z. X. Wu, "Experiment research on the evolution of cooperation and network game theory,"
Scientia Sinica (Physica, Mechanica and Astronomica, vol. 1, pp. 118-132, 2020, in Chinese.

[31] R. Esmaeilyfard, F. Hendessi, M. H. Manshaei et al., "A gametheoretic model for users' participation in ephemeral social vehicular networks," International Journal of Communication Systems, vol. 32, no. 12, Article ID e3998, 2019.

[32] L. Xu, C. Jiang, Y. Chen, Y. Ren, and K. J. R. Liu, "User participation in collaborative filtering-based recommendation systems: a game theoretic approach," IEEE Transactions on Cybernetics, vol. 49, no. 4, pp. 1339-1352, 2019.

[33] M. Liu, Y. Ma, Z. Liu, and X. You, “An IUR evolutionary game model on the patent cooperate of Shandong China," Physica A: Statistical Mechanics and Its Applications, vol. 475, no. 1, pp. 11-23, 2017.

[34] D. Friedman, "Evolutionary games in economics," Econometrica, vol. 59, no. 3, pp. 637-666, 1991.

[35] S. L. Johnson, S. Faraj, S. Faraj, and S. Kudaravalli, "Emergence of power laws in online communities: the role of social mechanisms and preferential attachment," MIS Quarterly, vol. 38, no. 3, pp. 795-808, 2014.

[36] C. Li and P. K. Maini, "An evolving network model with community structure," Journal of Physics A: Mathematical and General, vol. 38, no. 45, p. 9741, 2005.

[37] W. Liang, Y. Wang, Y. Shi, and G. Chen, "Co-occurrence network analysis of modern Chinese poems," Physica A: Statistical Mechanics and Its Applications, vol. 420, pp. 284293, 2015.

[38] Z.-X. Wu, J.-Y. Guan, X.-J. Xu, and Y.-H. Wang, "Evolutionary prisoner's dilemma game on Barabási-Albert scale-free networks," Physica A: Statistical Mechanics and Its Applications, vol. 379, no. 2, pp. 672-680, 2007.

[39] G. Szabo, J. Vukov, and A. Szolnoki, "Phase diagrams for an evolutionary prisoner's dilemma game on two-dimensional lattices," Physical Review E, vol. 72, Article ID 047107, 2005.

[40] D. Wu, H. Liu, Y. Bi, and H. Zhu, "Evolutionary game theoretic modeling and repetition of media distributed shared in P2P-based VANET," International Journal of Distributed Sensor Networks, vol. 10, no. 6, Article ID 718639, 2014.

[41] L. D. F. Costa, F. A. Rodrigues, G. Travieso, and P. R. Villas Boas, "Characterization of complex networks: a survey of measurements," Advances in Physics, vol. 56, no. 1, pp. 167-242, 2007.

[42] H.-J. Li, Z. Bu, Z. Wang, and J. Cao, "Dynamical clustering in electronic commerce systems via optimization and leadership expansion," IEEE Transactions on Industrial Informatics, vol. 16, no. 8, pp. 5327-5334, 2020.

[43] C. J. Cao, C. D. Li, Q. Yang, Y. Liu, and T. Qu, "A novel multiobjective programming model of relief distribution for sustainable disaster supply chain in large-scale natural disasters," Journal of Cleaner Production, vol. 174, pp. 1422-1435, 2018. 